Алгебра и анализ

Toм 21 (2009), № 3
St. Petersburg Math. J.

Vol. 21 (2010), No. 3, Pages 433-458 S 1061-0022(10)01102-7

Article electronically published on February 26, 2010

\title{
CLASSIFYING FINITE LOCALIZATIONS OF QUASICOHERENT SHEAVES
}

\author{
G. GARKUSHA
}

In memory of Vera Puninskaya

\begin{abstract}
Given a quasicompact, quasiseparated scheme $X$, a bijection between the tensor localizing subcategories of finite type in $\mathrm{Qcoh}(X)$ and the set of all subsets $Y \subseteq X$ of the form $Y=\bigcup_{i \in \Omega} Y_{i}$, with $X \backslash Y_{i}$ quasicompact and open for all $i \in \Omega$, is established. As an application, an isomorphism of ringed spaces

$$
\left(X, \mathcal{O}_{X}\right) \stackrel{\sim}{\longrightarrow}\left(\operatorname{spec}(\mathrm{Q} \operatorname{coh}(\mathrm{X})), \mathcal{O}_{\mathrm{Q} \operatorname{coh}(\mathrm{X})}\right)
$$

is constructed, where $\left(\operatorname{spec}(\mathrm{Q} \operatorname{coh}(\mathrm{X})), \mathcal{O}_{\mathrm{Q} \operatorname{coh}(\mathrm{X})}\right)$ is a ringed space associated with the lattice of tensor localizing subcategories of finite type. Also, a bijective correspondence between the tensor thick subcategories of perfect complexes $\mathcal{D}_{\text {per }}(X)$ and the tensor localizing subcategories of finite type in $\mathrm{Q} \operatorname{coh}(X)$ is established.
\end{abstract}

\section{§1. INTRODUCTION}

In his celebrated paper [1] on Abelian categories, Gabriel proved that for any Noetherian scheme $X$, the assignments

$$
\operatorname{coh} X \supseteq \mathcal{D} \mapsto \bigcup_{x \in \mathcal{D}} \operatorname{supp}_{X}(x) \quad \text { and } \quad X \supseteq U \mapsto\left\{x \in \operatorname{coh} X \mid \operatorname{supp}_{X}(x) \subseteq U\right\}
$$

induce bijections between

(1) the set of all tensor Serre subcategories of $\operatorname{coh} X$, and

(2) the set of all subsets $U \subseteq X$ of the form $U=\bigcup_{i \in \Omega} Y_{i}$, where, for all $i \in \Omega, Y_{i}$ has a quasicompact open complement $X \backslash Y_{i}$.

As a consequence of this result, $X$ can be reconstructed from its Abelian category, $\operatorname{coh} X$, of coherent sheaves (see Buan-Krause-Solberg [2, §8]). Garkusha and Prest [3]-[5] proved similar classification and reconstruction results for affine and projective schemes.

Given a quasicompact, quasiseparated scheme $X$, let $\mathcal{D}_{\text {per }}(X)$ denote the derived category of perfect complexes. It comes equipped with a tensor product $\otimes:=\otimes_{X}^{L}$. A thick triangulated subcategory $\mathcal{T}$ of $\mathcal{D}_{\text {per }}(X)$ is said to be a tensor subcategory if for every $E \in \mathcal{D}_{\text {per }}(X)$ and every object $A \in \mathcal{T}$, the tensor product $E \otimes A$ is also in $\mathcal{T}$. Thomason [ 6 ] established a classification similar to (1.1) for the tensor thick subcategories of $\mathcal{D}_{\text {per }}(X)$ in terms of the topology of $X$. Hopkins and Neeman (see [7, 8]) did the same in the case where $X$ is affine and Noetherian.

On the basis of Thomason's classification theorem, Balmer 9 reconstructed the Noetherian scheme $X$ out of the tensor thick triangulated subcategories of $\mathcal{D}_{\text {per }}(X)$. This result was generalized to quasicompact, quasiseparated schemes by Buan-Krause-Solberg [2].

2000 Mathematics Subject Classification. Primary 14A15, 18 F20.

Key words and phrases. Quasicompact, quasiseparated schemes, quasicoherent sheaves, localizing subcategories, thick subcategories. 
The main result of this paper is a generalization of the classification result by Garkusha and Prest [3]-[5] to schemes. Let $X$ be a quasicompact, quasiseparated scheme. We denote by $\mathrm{Q} \operatorname{coh}(X)$ the category of quasicoherent sheaves. We say that a localizing subcategory $\mathcal{S}$ of $\mathrm{Q} \operatorname{coh}(X)$ is of finite type if the canonical functor from the quotient category $\mathrm{Q} \operatorname{coh}(X) / \mathcal{S} \rightarrow \mathrm{Q} \operatorname{coh}(X)$ preserves the directed sums.

Theorem (Classification). Let $X$ be a quasicompact, quasiseparated scheme. Then the maps

$$
V \mapsto \mathcal{S}=\left\{\mathcal{F} \in \mathrm{Q} \operatorname{coh}(X) \mid \operatorname{supp}_{X}(\mathcal{F}) \subseteq V\right\}
$$

and

$$
\mathcal{S} \mapsto V=\bigcup_{\mathcal{F} \in \mathcal{S}} \operatorname{supp}_{X}(\mathcal{F})
$$

induce bijections between

(1) the set of all subsets of the form $V=\bigcup_{i \in \Omega} V_{i}$ with quasicompact open complement $X \backslash V_{i}$ for all $i \in \Omega$, and

(2) the set of all tensor localizing subcategories of finite type in $\mathrm{Qcoh}(X)$.

As an application of this classification theorem, we show that there is a one-to-one correspondence between the tensor localizing subcategories of finite type in $\mathrm{Qcoh}(X)$ and the tensor thick subcategories in $\mathcal{D}_{\text {per }}(X)$ (cf. [10, 3, 5] ).

Theorem. Let $X$ be a quasicompact and quasiseparated scheme. The assignments

$$
\mathcal{T} \mapsto \mathcal{S}=\left\{\mathcal{F} \in \operatorname{Qcoh}(X) \mid \operatorname{supp}_{X}(\mathcal{F}) \subseteq \bigcup_{n \in \mathbb{Z}, E \in \mathcal{T}} \operatorname{supp}_{X}\left(H_{n}(E)\right)\right\}
$$

and

$$
\mathcal{S} \mapsto\left\{E \in \mathcal{D}_{\text {per }}(X) \mid H_{n}(E) \in \mathcal{S} \text { for all } n \in \mathbb{Z}\right\}
$$

induce a bijection between

(1) the set of all tensor thick subcategories of $\mathcal{D}_{\text {per }}(X)$, and

(2) the set of all tensor localizing subcategories of finite type in $\mathrm{Qcoh}(X)$.

Another application of the classification theorem is the reconstruction theorem. A common approach in the noncommutative geometry is to study Abelian or triangulated categories and to think of them as the replacement of an underlying scheme. This idea goes back to work of Grothendieck and Manin. This approach is justified by the fact that a Noetherian scheme can be reconstructed from the Abelian category of coherent sheaves (Gabriel [1]) or by the category of perfect complexes (Balmer [9]). Rosenberg [1] proved that a quasicompact scheme $X$ is reconstructed from its category of quasicoherent sheaves.

In this paper we reconstruct a quasicompact, quasiseparated scheme $X$ from $\mathrm{Qcoh}(X)$. Our approach, similar to that used in [3, 4, 5], is entirely different from Rosenberg's [1] and is less abstract.

Following Buan-Krause-Solberg [2], we consider the lattice $L_{\mathrm{f} . \text { loc }, \otimes}(X)$ of tensor localizing subcategories of finite type in $\mathrm{Q} \operatorname{coh}(X)$, and also its prime ideal spectrum $\operatorname{Spec}(\mathrm{Q} \operatorname{coh}(X))$. In a natural way, this space is equipped with a sheaf of commutative rings $\mathcal{O}_{\mathrm{Q} \operatorname{coh}(X)}$. The following result says that the scheme $\left(X, \mathcal{O}_{X}\right)$ is isomorphic to $\left(\operatorname{Spec}(\mathrm{Q} \operatorname{coh}(X)), \mathcal{O}_{\mathrm{Q} \operatorname{coh}(X)}\right)$.

Theorem (Reconstruction). Let $X$ be a quasicompact and quasiseparated scheme. Then there is a natural isomorphism of ringed spaces

$$
f:\left(X, \mathcal{O}_{X}\right) \stackrel{\sim}{\longrightarrow}\left(\operatorname{Spec}(\mathrm{Q} \operatorname{coh}(X)), \mathcal{O}_{\mathrm{Q}} \operatorname{coh}(X)\right) .
$$


Among other results presented here, we mention Theorem 11, in which the finite localizations in a locally finitely presented Grothendieck category $\mathcal{C}$ are classified in terms of some topology on the injective spectrum $\mathrm{Sp} \mathcal{C}$; this generalizes a result of Herzog 12 and Krause [13] for locally coherent Grothendieck categories, and the classification and reconstruction theorems for coherent schemes.

\section{§2. Localization in Grothendieck Categories}

The category $\mathrm{Q} \operatorname{coh}(X)$ of quasicoherent sheaves over a scheme $X$ is a Grothendieck category (see [14]), so that we can apply the general localization theory for Grothendieck categories, which is of great utility in our analysis. For the reader's convenience we recall some basic facts of that theory.

We say that a subcategory $\mathcal{S}$ of an Abelian category $\mathcal{C}$ is a Serre subcategory if for any short exact sequence

$$
0 \rightarrow X \rightarrow Y \rightarrow Z \rightarrow 0
$$

in $\mathcal{C}$, the object $Y$ belongs to $\mathcal{S}$ if and only if $X, Z \in \mathcal{S}$. A Serre subcategory $\mathcal{S}$ of a Grothendieck category $\mathcal{C}$ is localizing if it is closed under taking direct limits. Equivalently, the inclusion functor $i: \mathcal{S} \rightarrow \mathcal{C}$ admits the right adjoint $t=t_{\mathcal{S}}: \mathcal{C} \rightarrow \mathcal{S}$ that takes every object $X \in \mathcal{C}$ to the maximal subobject $t(X)$ of $X$ belonging to $\mathcal{S}$. The functor $t$ is called the torsion functor. An object $C$ of $\mathcal{C}$ is said to be $\mathcal{S}$-torsion free if $t(C)=0$. Given a localizing subcategory $\mathcal{S}$ of $\mathcal{C}$, the quotient category $\mathcal{C} / \mathcal{S}$ consists of $C \in \mathcal{C}$ such that $t(C)=t^{1}(C)=0$. The objects in $\mathcal{C} / \mathcal{S}$ are called $\mathcal{S}$-closed objects. For any $C \in \mathcal{C}$, there exists a canonical exact sequence

$$
0 \rightarrow A^{\prime} \rightarrow C \stackrel{\lambda_{C}}{\longrightarrow} C_{\mathcal{S}} \rightarrow A^{\prime \prime} \rightarrow 0
$$

where $A^{\prime}=t(C), A^{\prime \prime} \in \mathcal{S}$, and $C_{\mathcal{S}} \in \mathcal{C} / \mathcal{S}$ is the maximal essential extension of $\widetilde{C}=C / t(C)$ such that $C_{\mathcal{S}} / \widetilde{C} \in \mathcal{S}$. The object $C_{\mathcal{S}}$ is determined uniquely up to a canonical isomorphism and is called the $\mathcal{S}$-envelope of $C$. Moreover, the inclusion functor $i: \mathcal{C} / \mathcal{S} \rightarrow \mathcal{C}$ has a left adjoint localizing functor $(-)_{\mathcal{S}}: \mathcal{C} \rightarrow \mathcal{C} / \mathcal{S}$, which is also exact. It takes each $C \in \mathcal{C}$ to $C_{\mathcal{S}} \in \mathcal{C} / \mathcal{S}$. Then,

$$
\operatorname{Hom}_{\mathcal{C}}(X, Y) \cong \operatorname{Hom}_{\mathcal{C} / \mathcal{S}}\left(X_{\mathcal{S}}, Y\right)
$$

for all $X \in \mathcal{C}$ and $Y \in \mathcal{C} / \mathcal{S}$.

If $\mathcal{C}$ and $\mathcal{D}$ are Grothendieck categories, $q: \mathcal{C} \rightarrow \mathcal{D}$ is an exact functor, and a functor $s: \mathcal{D} \rightarrow \mathcal{C}$ is fully faithful and right adjoint to $q$, then $\mathcal{S}:=\operatorname{Ker} q$ is a localizing subcategory and there exists an equivalence $\mathcal{C} / \mathcal{S} \cong \stackrel{H}{\cong} \mathcal{D}$ such that $H \circ(-)_{\mathcal{S}}=q$. We shall refer to the pair $(q, s)$ as a localization pair.

The following result is an example of a localization pair.

Proposition 1 (cf. 1, §III.5; Proposition VI.3]). Let $X$ be a scheme, and let $U$ be an open subset of $X$ such that the canonical injection $j: U \rightarrow X$ is a quasicompact map. Then $j_{*}(\mathcal{G})$ is a quasicoherent $\mathcal{O}_{X}$-module for any quasicoherent $\left.\mathcal{O}_{X}\right|_{U}$-module $\mathcal{G}$, and the pair of adjoint functors $\left(j^{*}, j_{*}\right)$ is a localization pair. That is, the category of quasicoherent $\left.\mathcal{O}_{X}\right|_{U}$-modules $\mathrm{Q} \operatorname{coh}(U)$ is equivalent to $\mathrm{Qcoh}(X) / \mathcal{S}$, where $\mathcal{S}=\operatorname{Ker} j^{*}$. Moreover, a quasicoherent $\mathcal{O}_{X}$-module $\mathcal{F}$ belongs to the localizing subcategory $\mathcal{S}$ if and only if $\operatorname{supp}_{X}(\mathcal{F})=\left\{P \in X \mid \mathcal{F}_{P} \neq 0\right\} \subseteq Z=X \backslash U$. Also, for any $\mathcal{F} \in \operatorname{Qcoh}(X)$ we have $t_{\mathcal{S}}(\mathcal{F})=\mathcal{H}_{Z}^{0}(\mathcal{F})$, where $\mathcal{H}_{Z}^{0}(\mathcal{F})$ stands for the subsheaf of $\mathcal{F}$ with supports in $Z$.

Proof. The fact that $j_{*}(\mathcal{G})$ is a quasicoherent $\mathcal{O}_{X}$-module follows from [15, I.6.9.2]. Clearly, the functor $j^{*}:\left.\mathcal{F} \mapsto \mathcal{F}\right|_{U}$ is exact, $\left.j_{*}(\mathcal{G})\right|_{U}=j^{*} j_{*}(\mathcal{G})=\mathcal{G}$ by [15, I.6.9.2]. It follows that $j_{*}$ is fully faithful, and hence $\left(j^{*}, j_{*}\right)$ is a localization pair. 
The fact that $\mathcal{F} \in \mathcal{S}$ if and only if $\operatorname{supp}_{X}(\mathcal{F}) \subseteq Z$ is obvious. Finally, by 16, Ex. II.1.20] we have an exact sequence

$$
0 \rightarrow \mathcal{H}_{Z}^{0}(\mathcal{F}) \rightarrow \mathcal{F} \stackrel{\rho_{\mathcal{F}}}{\longrightarrow} j_{*} j^{*}(\mathcal{F}) .
$$

Since the morphism $\rho_{\mathcal{F}}$ can be regarded as an $\mathcal{S}$-envelope for $\mathcal{F}$, we see that $\operatorname{Ker} \rho_{\mathcal{F}}=$ $t_{\mathcal{S}}(\mathcal{F})=\mathcal{H}_{Z}^{0}(\mathcal{F})$.

Given a subcategory $\mathcal{X}$ of a Grothendieck category $\mathcal{C}$, we denote by $\sqrt{ } \mathcal{X}$ the smallest localizing subcategory of $\mathcal{C}$ containing $\mathcal{X}$. To describe $\sqrt{ } \mathcal{X}$ intrinsically, we need the notion of a subquotient.

Definition. Given objects $A, B \in \mathcal{C}$, we say that $A$ is a subquotient of $B$, or $A \prec B$, if there is a filtration of $B$ by subobjects $B=B_{0} \geq B_{1} \geq B_{2} \geq 0$ such that $A \cong B_{1} / B_{2}$. In other words, $A$ is isomorphic to a subobject of a quotient object of $B$.

Given a subcategory $\mathcal{X}$ of $\mathcal{C}$, we denote by $\langle\mathcal{X}\rangle$ the full subcategory of subquotients of objects from $\mathcal{X}$. Clearly, $\langle\mathcal{X}\rangle=\langle\langle\mathcal{X}\rangle\rangle$ because the relation $A \prec B$ is transitive, and $\mathcal{X}=\langle\mathcal{X}\rangle$ if and only if $\mathcal{X}$ is closed under subobjects and quotient objects. If $\mathcal{X}$ is closed under direct sums, then so is $\langle\mathcal{X}\rangle$.

Proposition 2. For any subcategory $\mathcal{X}$ of a Grothendieck category $\mathcal{C}$, an object $X$ belongs to $\sqrt{ } \mathcal{X}$ if and only if there is a filtration

$$
X_{0} \subset X_{1} \subset \cdots \subset X_{\beta} \subset \cdots
$$

such that $X=\bigcup_{\beta} X_{\beta}, X_{\gamma}=\bigcup_{\beta<\gamma} X_{\beta}$ if $\gamma$ is a limit ordinal, and $X_{0}, X_{\beta+1} / X_{\beta} \in\left\langle\mathcal{X}^{\oplus}\right\rangle$ for any $\beta$, where $\mathcal{X}^{\oplus}$ stands for the subcategory of $\mathcal{C}$ consisting of direct sums of objects in $\mathcal{X}$.

Proof. It is easily seen that every object having such a filtration belongs to $\sqrt{ } \mathcal{X}$. It suffices to show that the full subcategory $\mathcal{S}$ of such objects is localizing. Let

$$
X \longmapsto Y \stackrel{g}{\rightarrow} Z
$$

be a short exact sequence with $X, Z \in \mathcal{S}$. Let $X_{0} \subset \cdots \subset X_{\beta} \subset \cdots$ and $Z_{0} \subset \cdots \subset$ $Z_{\alpha} \subset \cdots$ be the corresponding filtrations. Put $Y_{\alpha}=g^{-1}\left(Z_{\alpha}\right)$. Then, for any $\alpha$, we have a short exact sequence

$$
X \longmapsto Y_{\alpha} \stackrel{g_{\alpha}}{\rightarrow} Z_{\alpha}
$$

with $Y_{\alpha+1} / Y_{\alpha} \cong Z_{\alpha+1} / Z_{\alpha}$. Let the filtrations for $X$ and $Z$ be indexed by well-ordered sets $\delta$ and $\gamma$, respectively. Then we have the following filtration for $Y$ indexed by the well-ordered set $\delta+\gamma$ :

$$
X_{0} \subset \cdots \subset X_{\beta} \subset \cdots \subset X=\bigcup_{\beta} X_{\beta} \subset Y_{0} \subset \cdots \subset Y_{\alpha} \subset \cdots .
$$

It follows that $Y \in \mathcal{S}$. We see that $\mathcal{S}$ is closed under extensions.

Now, let $Y \in \mathcal{S}$ with a filtration $Y_{0} \subset \cdots \subset Y_{\alpha} \subset \cdots$. Set $X_{\alpha}=X \cap Y_{\alpha}$ and $Z_{\alpha}=Y_{\alpha} / X_{\alpha}$. We get filtrations $X_{0} \subset \cdots \subset X_{\alpha} \subset \cdots$ and $Z_{0} \subset \cdots \subset Z_{\alpha} \subset \cdots$ for $X$ and $Z$, respectively. Thus, $X, Z \in \mathcal{S}$, so that $\mathcal{S}$ is a Serre subcategory. Clearly, it is closed under direct sums; hence it is localizing.

Corollary 3. Let $\mathcal{X}$ be a subcategory in $\mathcal{C}$ closed under subobjects, quotient objects, and direct sums. An object $M \in \mathcal{C}$ is $\sqrt{ } \mathcal{X}$-closed if and only if $\operatorname{Hom}(X, M)=\operatorname{Ext}^{1}(X, M)=0$ for all $X \in \mathcal{X}$. 
Proof. Suppose $\operatorname{Hom}(X, M)=\operatorname{Ext}^{1}(X, M)=0$ for all $X \in \mathcal{X}$. We need to check that $\operatorname{Hom}(Y, M)=\operatorname{Ext}^{1}(Y, M)=0$ for all $Y \in \sqrt{ } \mathcal{X}$. By Proposition 2, there is a filtration

$$
Y_{0} \subset Y_{1} \subset \cdots \subset Y_{\beta} \subset \cdots
$$

such that $Y=\bigcup_{\beta} Y_{\beta}, Y_{\gamma}=\bigcup_{\beta<\gamma} Y_{\beta}$ if $\gamma$ is a limit ordinal, and $Y_{0}, Y_{\beta+1} / Y_{\beta} \in\left\langle\mathcal{X}^{\oplus}\right\rangle=\mathcal{X}$. For any $\beta$, we have an exact sequence

$$
\begin{aligned}
\operatorname{Hom}\left(Y_{\beta+1} / Y_{\beta}, M\right) & \rightarrow \operatorname{Hom}\left(Y_{\beta+1}, M\right) \rightarrow \operatorname{Hom}\left(Y_{\beta}, M\right) \\
& \rightarrow \operatorname{Ext}^{1}\left(Y_{\beta+1} / Y_{\beta}, M\right) \rightarrow \operatorname{Ext}^{1}\left(Y_{\beta+1}, M\right) \rightarrow \operatorname{Ext}^{1}\left(Y_{\beta}, M\right) .
\end{aligned}
$$

Hence, if $\operatorname{Hom}\left(Y_{\beta}, M\right)=\operatorname{Ext}^{1}\left(Y_{\beta}, M\right)=0$, then $\operatorname{Hom}\left(Y_{\beta+1}, M\right)=\operatorname{Ext}^{1}\left(Y_{\beta+1}, M\right)=0$ because $Y_{\beta+1} / Y_{\beta} \in \mathcal{X}$. Since $Y_{0} \in \mathcal{X}$, it follows that $\operatorname{Hom}\left(Y_{\beta}, M\right)=\operatorname{Ext}^{1}\left(Y_{\beta}, M\right)=0$ for all finite $\beta$.

Let $\gamma$ be a limit ordinal, and let $\operatorname{Hom}\left(Y_{\beta}, M\right)=\operatorname{Ext}^{1}\left(Y_{\beta}, M\right)=0$ for all $\beta<\gamma$. We have $\operatorname{Hom}\left(Y_{\gamma}, M\right)=\lim _{\beta<\gamma} \operatorname{Hom}\left(Y_{\beta}, M\right)=0$. To show that $\operatorname{Ext}^{1}\left(Y_{\gamma}, M\right)=0$, we need to prove that every short exact sequence

$$
M \longmapsto N \stackrel{p}{\rightarrow} Y_{\gamma}
$$

is split. There is a commutative diagram

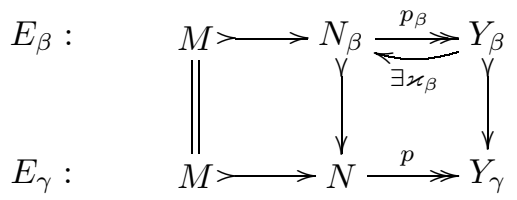

with $N_{\beta}=p^{-1}\left(Y_{\beta}\right)$. Clearly, $E_{\gamma}=\bigcup_{\beta} E_{\beta}$. Since the upper row splits, there exists a morphism $\varkappa_{\beta}$ such that $p_{\beta} \varkappa_{\beta}=1$. Consider the following commutative diagram:

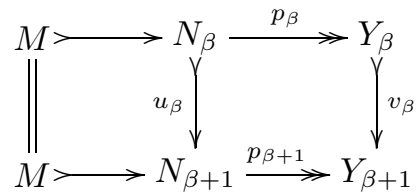

We want to check that $\varkappa_{\beta+1} v_{\beta}=u_{\beta} \varkappa_{\beta}$. Since the right square is Cartesian and $p_{\beta+1} \varkappa_{\beta+1} v_{\beta}=v_{\beta}$, there exists a unique morphism $\tau: Y_{\beta} \rightarrow N_{\beta}$ such that $p_{\beta} \tau=1$ and $u_{\beta} \tau=\varkappa_{\beta+1} v_{\beta}$. We claim that $\tau=\varkappa_{\beta}$. Indeed, $p_{\beta}\left(\tau-\varkappa_{\beta}\right)=0$, whence $\tau-\varkappa_{\beta}$ factors through $M$. This is possible only if $\tau-\varkappa_{\beta}=0$, because $\operatorname{Hom}\left(Y_{\beta}, M\right)=0$ by assumption. Therefore, $\tau=\varkappa_{\beta}$. It follows that the family of morphisms $\varkappa_{\beta}: Y_{\beta} \rightarrow N_{\beta}$ is directed and then $p \circ\left(\lim _{\longrightarrow} \varkappa_{\beta}\right)=\left(\lim _{\longrightarrow} p_{\beta}\right) \circ\left(\underline{\lim } \varkappa_{\beta}\right)=\lim _{\longrightarrow}\left(p_{\beta} \varkappa_{\beta}\right)=1$. Thus, $p$ is split.

Recall that the injective spectrum or the Gabriel spectrum $\mathrm{SpC}$ of a Grothendieck category $\mathcal{C}$ is the set of isomorphism classes of indecomposable injective objects in $\mathcal{C}$. It plays an important role in our analysis. Given a subcategory $\mathcal{X}$ in $\mathcal{C}$, we denote

$$
(\mathcal{X})=\left\{E \in \operatorname{Sp} \mathcal{C} \mid \operatorname{Hom}_{\mathcal{C}}(X, E) \neq 0 \text { for some } X \in \mathcal{X}\right\} .
$$

Using Proposition 2 and the fact that the functor $\operatorname{Hom}(-, E), E \in \mathrm{Sp} \mathcal{C}$, is exact, we see that $(\mathcal{X})=\bigcup_{X \in \mathcal{X}}(X)=(\sqrt{ } \mathcal{X})$.

Proposition 4. The collection

$$
\{(\mathcal{S}) \mid \mathcal{S} \subset \mathcal{C} \text { is a localizing subcategory }\}
$$


of subsets of $\mathrm{SpC}$ satisfies the axioms for the open sets of a topology on the injective spectrum $\mathrm{Sp} \mathcal{C}$. This topological space will be denoted by $\mathrm{Sp}_{\text {gab }} \mathcal{C}$. Moreover, the map

$$
\mathcal{S} \longmapsto(\mathcal{S})
$$

is an inclusion-preserving bijection between the localizing subcategories $\mathcal{S}$ of $\mathcal{C}$ and the open subsets of $\mathrm{Sp}_{\mathrm{gab}} \mathcal{C}$.

Proof. First, note that $(0)=\varnothing$ and $(\mathcal{C})=\operatorname{Sp} \mathcal{C}$. We have $\left(\mathcal{S}_{1}\right) \cap\left(\mathcal{S}_{2}\right)=\left(\mathcal{S}_{1} \cap \mathcal{S}_{2}\right)$ because every $E \in \operatorname{SpC}$ is uniform and $0 \neq t_{\mathcal{S}_{1}}(E) \cap t_{\mathcal{S}_{2}}(E) \in \mathcal{S}_{1} \cap \mathcal{S}_{2}$ whenever $E \in\left(\mathcal{S}_{1}\right) \cap\left(\mathcal{S}_{2}\right)$. Also, $\bigcup_{i \in I}\left(\mathcal{S}_{i}\right)=\left(\bigcup_{i \in I} \mathcal{S}_{i}\right)=\left(\sqrt{ } \bigcup_{i \in I} \mathcal{S}_{i}\right)$.

Clearly, the map (2.1) is bijective, because every localizing subcategory $\mathcal{S}$ consists precisely of the objects $X$ such that $\operatorname{Hom}(X, E)=0$ for all $E \in \operatorname{Sp} \mathcal{C} \backslash(\mathcal{S})$.

For a localizing subcategory $\mathcal{S}$ in $\mathcal{C}$, the injective spectrum $\operatorname{Sp}_{\text {gab }}(\mathcal{C} / \mathcal{S})$ can be viewed as the closed subset $\mathrm{Sp}_{\text {gab }} \mathcal{C} \backslash(\mathcal{S})$. Moreover, the inclusion

$$
\mathrm{Sp}_{\text {gab }}(\mathcal{C} / \mathcal{S}) \hookrightarrow \mathrm{Sp}_{\text {gab }} \mathcal{C}
$$

is a closed map. Indeed, if $U$ is a closed subset in $\operatorname{Sp}_{\text {gab }}(\mathcal{C} / \mathcal{S})$, then there is a unique localizing subcategory $\mathcal{T}$ in $\mathcal{C} / \mathcal{S}$ such that $U=\operatorname{Sp}_{\text {gab }}(\mathcal{C} / \mathcal{S}) \backslash(\mathcal{T})$. By [17, 1.7] there is a unique localizing subcategory $\mathcal{P}$ in $\mathcal{C}$ containing $\mathcal{S}$ and such that $\mathcal{C} / \mathcal{P}$ is equivalent to $(\mathcal{C} / \mathcal{S}) / \mathcal{T}$. It follows that $U=\mathrm{Sp}_{\text {gab }} \mathcal{C} \backslash(\mathcal{P})$, whence $U$ is closed in $\mathrm{Sp}_{\text {gab }} \mathcal{C}$.

On the other hand, let $\mathcal{Q}$ be a localizing subcategory of $\mathcal{C}$. We show that $O:=$ $(\mathcal{Q}) \cap \mathrm{Sp}_{\text {gab }}(\mathcal{C} / \mathcal{S})$ is an open subset in $\operatorname{Sp}_{\text {gab }}(\mathcal{C} / \mathcal{S})$.

Lemma 5. Let $\widehat{\mathcal{Q}}$ denote the full subcategory of objects of the form $X_{\mathcal{S}}$ with $X \in \mathcal{Q}$. Then $\widehat{\mathcal{Q}}$ is closed under direct sums, subobjects, and quotient objects in $\mathcal{C} / \mathcal{S}$, and $O=(\sqrt{ } \widehat{\mathcal{Q}})$. Moreover, if $\mathcal{T}$ is a unique localizing subcategory of $\mathcal{C}$ containing $\mathcal{S}$ such that $\mathcal{C} / \mathcal{T} \cong$ $(\mathcal{C} / \mathcal{S}) / \sqrt{ } \mathcal{Q}$, then

$$
\mathcal{T}=\sqrt{ }(\mathcal{Q} \cup \mathcal{S})
$$

i.e., $\mathcal{T}$ is the smallest localizing subcategory containing $\mathcal{Q}$ and $\mathcal{S}$. We shall also refer to $\mathcal{T}$ as the join of $\mathcal{Q}$ and $\mathcal{S}$.

Proof. First, we prove that $\widehat{\mathcal{Q}}$ is closed under direct sums, subobjects, and quotient objects in $\mathcal{C} / \mathcal{S}$. Clearly, it is closed under direct sums. Let $Y$ be a subobject of $X_{\mathcal{S}}$, $X \in \mathcal{Q}$, and let $\lambda_{X}: X \rightarrow X_{\mathcal{S}}$ be the $\mathcal{S}$-envelope for $X$. Then $W=\lambda_{X}^{-1}(Y)$ is a subobject of $X$, so that it belongs to $\mathcal{Q}$, and $Y=W_{\mathcal{S}}$. If $Z$ is a $\mathcal{C} / \mathcal{S}$-quotient of $X_{\mathcal{S}}$ and $\pi: X_{\mathcal{S}} \rightarrow Z$ is the canonical projection, then $Z=V_{\mathcal{S}}$ with $V=X / \operatorname{Ker}\left(\pi \lambda_{X}\right) \in \mathcal{Q}$. So, $\widehat{\mathcal{Q}}$ is also closed under subobjects and quotient objects in $\mathcal{C} / \mathcal{S}$.

It follows that $\left\langle\widehat{\mathcal{Q}}^{\oplus}\right\rangle=\widehat{\mathcal{Q}}$ and $(\widehat{\mathcal{Q}})=(\sqrt{ } \widehat{\mathcal{Q}})$. On the other hand, it is easily seen that $O=(\hat{\mathcal{Q}})$. Thus, $O$ is open in $\operatorname{Sp}_{\text {gab }}(\mathcal{C} / \mathcal{S})$.

Clearly,

$$
(\mathcal{T})=(\mathcal{Q}) \cup(\mathcal{S})=(\mathcal{Q} \cup \mathcal{S})=(\sqrt{ }(\mathcal{Q} \cup \mathcal{S}))
$$

By Proposition 4, we have $\mathcal{T}=\sqrt{ }(\mathcal{Q} \cup \mathcal{S})$.

We summarize the above arguments as follows.

Proposition 6. For any localizing subcategory $\mathcal{S}$ in $\mathcal{C}$, the topology on $\mathrm{Sp}_{\text {gab }}(\mathcal{C} / \mathcal{S}) c o-$ incides with the subspace topology induced by $\mathrm{Sp}_{\text {gab }} \mathcal{C}$. 


\section{§3. Finite localizations of Grothendieck Categories}

Mostly, in this paper we are interested in finite localizations of a Grothendieck category $\mathcal{C}$. For this, we should impose some finiteness conditions on $\mathcal{C}$.

Recall that an object $X$ of a Grothendieck category $\mathcal{C}$ is finitely generated if whenever there are subobjects $X_{i} \subseteq X$ with $i \in I$ satisfying $X=\sum_{i \in I} X_{i}$, there is a finite subset $J \subset I$ such that $X=\sum_{i \in J} X_{i}$. The full subcategory of finitely generated objects is denoted by $\operatorname{fg} \mathcal{C}$. A finitely generated object $X$ is said to be finitely presented if every epimorphism $\gamma: Y \rightarrow X$ with $Y \in$ fg $\mathcal{C}$ has finitely generated kernel $\operatorname{Ker} \gamma$. By fp $\mathcal{C}$ we denote the full subcategory consisting of finitely presented objects. The category $\mathcal{C}$ is locally finitely presented if every object $C \in \mathcal{C}$ is a direct $\operatorname{limit} C=\lim C_{i}$ of finitely presented objects $C_{i}$, or equivalently, if $\mathcal{C}$ possesses a family of finitely presented generators. In such a category, every finitely generated object $A \in \mathcal{C}$ admits an epimorphism $\eta: B \rightarrow A$ from a finitely presented object $B$. Finally, we refer to a finitely presented object $X \in \mathcal{C}$ as coherent if every finitely generated subobject of $X$ is finitely presented. The corresponding full subcategory of coherent objects will be denoted by $\operatorname{coh} \mathcal{C}$. A locally finitely presented category $\mathcal{C}$ is locally coherent if $\operatorname{coh} \mathcal{C}=\mathrm{fp} \mathcal{C}$. Obviously, a locally finitely presented category $\mathcal{C}$ is locally coherent if and only if $\operatorname{coh} \mathcal{C}$ is an Abelian category.

In [14] it was shown that the category of quasicoherent sheaves $Q \operatorname{coh}(X)$ over a scheme $X$ is a locally $\lambda$-presentable category, where $\lambda$ is a certain regular cardinal. However, for some nice schemes, most often used in practice in algebraic geometry, such as quasicompact and quasiseparated, there are sufficiently many finitely presented generators for $\operatorname{Qcoh}(X)$.

Proposition 7. Let $X$ be a quasicompact and quasiseparated scheme. Then $\mathrm{Qcoh}(X)$ is a locally finitely presented Grothendieck category. An object $\mathcal{F}$ belongs to $\operatorname{fp}(\mathrm{Qcoh}(X))$ if and only if it is locally finitely presented.

Proof. By [15, I.6.9.12], every quasicoherent sheaf is a direct limit of locally finitely presented sheaves. From [18, Proposition 75] it follows that the locally finitely presented sheaves are precisely the finitely presented objects in $\mathrm{Qcoh}(X)$.

Recall that a localizing subcategory $\mathcal{S}$ of a Grothendieck category $\mathcal{C}$ is of finite type (respectively, of strictly finite type) if the functor $i: \mathcal{C} / \mathcal{S} \rightarrow \mathcal{C}$ preserves directed sums (respectively, direct limits). If $\mathcal{C}$ is a locally finitely generated (respectively, locally finitely presented) Grothendieck category and $\mathcal{S}$ is of finite type (respectively, of strictly finite type), then $\mathcal{C} / \mathcal{S}$ is a locally finitely generated (respectively, locally finitely presented) Grothendieck category, and

$$
\left.\operatorname{fg}(\mathcal{C} / \mathcal{S})=\left\{C_{\mathcal{S}} \mid C \in \operatorname{fg} \mathcal{C}\right\} \quad \text { (respectively, } \operatorname{fp}(\mathcal{C} / \mathcal{S})=\left\{C_{\mathcal{S}} \mid C \in \mathrm{fp} \mathcal{C}\right\}\right)
$$

If $\mathcal{C}$ is a locally coherent Grothendieck category, then $\mathcal{S}$ is of finite type if and only if it is of strictly finite type (see, e.g., [17, 5.14]). In this case $\mathcal{C} / \mathcal{S}$ is locally coherent.

The following proposition says that the localizing subcategories of finite type in a locally finitely presented Grothendieck category $\mathcal{C}$ are completely determined by finitely presented torsion objects (cf. [12, 13]).

Proposition 8. Let $\mathcal{S}$ be a localizing subcategory of finite type in a locally finitely presented Grothendieck category $\mathcal{C}$. Then

$$
\mathcal{S}=\sqrt{ }(\operatorname{fp} \mathcal{C} \cap \mathcal{S})
$$

Proof. Obviously, $\sqrt{ }(\operatorname{fp} \mathcal{C} \cap \mathcal{S}) \subset \mathcal{S}$. Let $X \in \mathcal{S}$, and let $Y$ be a finitely generated subobject of $X$. There is an epimorphism $\eta: Z \rightarrow Y$ with $Z \in$ fp $\mathcal{C}$. By [17, 5.8], there is a finitely generated subobject $W \subset \operatorname{Ker} \eta$ such that $Z / W \in \mathcal{S}$. It follows that $Z / W \in \operatorname{fp} \mathcal{C} \cap \mathcal{S}$ and $Y$ is an epimorphic image of $Z / W$. Since $X$ is a direct union of finitely generated 
torsion subobjects, we see that $X$ is an epimorphic image of some $\bigoplus_{i \in I} S_{i}$ with each $S_{i}$ in $\operatorname{fp} \mathcal{C} \cap \mathcal{S}$. Therefore, $\mathcal{S} \subset \sqrt{ }(\operatorname{fp} \mathcal{C} \cap \mathcal{S})$.

Lemma 9. Let $\mathcal{Q}$ and $\mathcal{S}$ be two localizing subcategories in a Grothendieck category $\mathcal{C}$. If $X \in \mathcal{C}$ is both $\mathcal{Q}$-closed and $\mathcal{S}$-closed, then it is $\mathcal{T}=\sqrt{ }(\mathcal{Q} \cup \mathcal{S})$-closed.

Proof. By Lemma $5 \mathcal{C} / \mathcal{T} \cong(\mathcal{C} / \mathcal{S}) / \sqrt{ } \widehat{\mathcal{Q}}$, where $\widehat{\mathcal{Q}}=\left\{C_{\mathcal{S}} \in \mathcal{C} / \mathcal{S} \mid C \in \mathcal{Q}\right\}$ and $\widehat{\mathcal{Q}}$ is closed under direct sums, subobjects, and quotient objects in $\mathcal{C} / \mathcal{S}$. To show that $X=X_{\mathcal{S}}$ is a $\mathcal{T}$-closed object it suffices to check that $X$ is $\sqrt{ } \hat{\mathcal{Q}}$-closed in $\mathcal{C} / \mathcal{S}$. Obviously, $\operatorname{Hom}_{\mathcal{C} / \mathcal{S}}(A, X)=0$ for all $A \in \widehat{\mathcal{Q}}$.

Consider a short exact sequence

$$
E: X \longmapsto Y \stackrel{p}{\rightarrow} C_{\mathcal{S}}
$$

in $\mathcal{C} / \mathcal{S}$, where $C \in \mathcal{Q}$. One can construct a commutative diagram

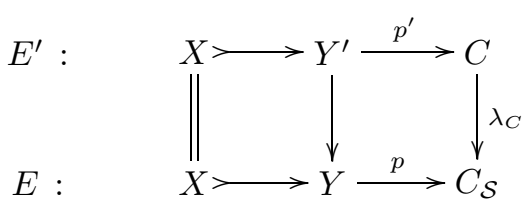

in $\mathcal{C}$, where the right square is Cartesian. Put $C^{\prime}=\operatorname{Im}_{\mathcal{C}} p^{\prime}$; then $C^{\prime} \in \mathcal{Q}, C_{\mathcal{S}}^{\prime}=C_{\mathcal{S}}$, and the short exact sequence

$$
E^{\prime \prime}: X \longmapsto Y^{\prime} \stackrel{p^{\prime}}{\rightarrow} C^{\prime}
$$

splits because $\operatorname{Ext}_{\mathcal{C}}^{1}\left(C^{\prime}, X\right)=0$. It follows that $E$ splits for $E=E_{\mathcal{S}}^{\prime}=E_{\mathcal{S}}^{\prime \prime}$. Therefore, $X$ is $\sqrt{ } \widehat{\mathcal{Q}}$-closed by Corollary 3

Below we shall need the following statement.

Lemma 10. For any family of localizing subcategories of finite type $\left\{\mathcal{S}_{i}\right\}_{i \in I}$ in a locally finitely presented Grothendieck category $\mathcal{C}$, their join $\mathcal{T}=\sqrt{ }\left(\bigcup_{i \in I} \mathcal{S}_{i}\right)$ is a localizing subcategory of finite type.

Proof. First, we consider the case where $I$ is finite. It suffices to show that the join $\mathcal{T}=\sqrt{ }(\mathcal{Q} \cup \mathcal{S})$ of two localizing subcategories $\mathcal{Q}$ and $\mathcal{S}$ of finite type is of finite type. We must check that the inclusion functor $\mathcal{C} / \mathcal{T} \rightarrow \mathcal{C}$ respects directed sums. Clearly, it suffices to verify that $X=\sum_{\mathcal{C}} X_{\alpha}$ is a $\mathcal{T}$-closed object whenever all $X_{\alpha}$ 's are $\mathcal{T}$-closed. Since $\mathcal{Q}$ and $\mathcal{S}$ are of finite type, $X$ is both $\mathcal{Q}$-closed and $\mathcal{S}$-closed. Lemma 9 implies that $X$ is $\mathcal{T}$-closed. Therefore, $\mathcal{T}$ is of finite type.

Now, let $\left\{\mathcal{S}_{i}\right\}_{i \in I}$ be an arbitrary set of localizing subcategories of finite type. Without loss of generality we may assume that $I$ is a directed set and $\mathcal{S}_{i} \subset \mathcal{S}_{j}$ for $i \leq j$. Indeed, given a finite subset $J \subset I$, denote by $\mathcal{S}_{J}$ the localizing subcategory of finite type $\sqrt{ }\left(\bigcup_{j \in J} \mathcal{S}_{j}\right)$. Then, obviously, the set $R$ of all finite subsets $J$ of $I$ is directed, $\mathcal{S}_{J} \subset \mathcal{S}_{J^{\prime}}$ for any $J \subset J^{\prime}$, and $\mathcal{T}=\sqrt{ }\left(\bigcup_{J \in R} \mathcal{S}_{J}\right)$.

Let $\mathcal{X}$ denote the full subcategory of $\mathcal{C}$ of the objects that can be presented as directed sums $\sum X_{\alpha}$ with each $X_{\alpha}$ in $\bigcup_{i \in I} \mathcal{S}_{i}$. Since $I$ is a directed set and $\mathcal{S}_{i} \subset \mathcal{S}_{j}$ for $i \leq j$, it follows that a direct sum $X=\bigoplus_{\gamma \in \Gamma} X_{\gamma}$ with each $X_{\gamma}$ in $\bigcup_{i \in I} \mathcal{S}_{i}$ is in $\mathcal{X}$. Indeed, $X=\sum X_{S}$ with $S$ running through all finite subsets of $\Gamma$ and $X_{S}=\bigoplus_{\gamma \in S} X_{\gamma} \in \bigcup_{i \in I} \mathcal{S}_{i}$. Therefore, if $\left\{X_{\beta}\right\}_{\beta \in B}$ is a family of subobjects of an object $X$ and each $X_{\beta}$ belongs to $\bigcup_{i \in I} \mathcal{S}_{i}$, then the direct union $\sum X_{\beta}$ belongs to $\mathcal{X}$.

The subcategory $\mathcal{X}$ is closed under subobjects and quotient objects. Indeed, let $X=$ $\sum X_{\alpha}$ with each $X_{\alpha}$ in $\bigcup_{i \in I} \mathcal{S}_{i}$. Consider a short exact sequence

$$
Y \longmapsto X \rightarrow Z \text {. }
$$


We set $Y_{\alpha}=Y \cap X_{\alpha}$ and $Z_{\alpha}=X_{\alpha} / Y_{\alpha} \subset Z$. Then both $Y_{\alpha}$ and $Z_{\alpha}$ are in $\bigcup_{i \in I} \mathcal{S}_{i}$, $Y=Y \cap\left(\sum X_{\alpha}\right)=\sum Y \cap X_{\alpha}=\sum Y_{\alpha}$, and $Z=\sum Z_{\alpha}$. So, $Y, Z \in \mathcal{X}$.

Clearly, $\mathcal{X}$ is closed under directed sums, in particular, under direct sums, whence $\mathcal{X}=\left\langle\mathcal{X}^{\oplus}\right\rangle$ and $\mathcal{T}=\sqrt{ } \mathcal{X}$. If we show that every direct limit $C=\lim C_{\delta}$ of $\mathcal{T}$-closed objects $C_{\delta}$ has no $\mathcal{T}$-torsion, then from [17, 5.8] it will follow that $\mathcal{T}$ is of finite type.

Proposition 2 shows that it suffices to check that $\operatorname{Hom}_{\mathcal{C}}(X, C)=0$ for any object $X \in \mathcal{X}$. Let $Y$ be a finitely generated subobject in $X$. There is an index $i_{0} \in I$ with $Y \in \mathcal{S}_{i_{0}}$, and an epimorphism $\eta: Z \rightarrow Y$ with $Z \in$ fp $\mathcal{C}$. By [17, 5.8], there exists a finitely generated subobject $W$ of $\operatorname{Ker} \eta$ such that $Z / W \in \mathcal{S}_{i_{0}}$. Since $Z / W \in \operatorname{fp} \mathcal{C}$, we have $\operatorname{Hom}(Z / W, C)=\underset{\lim }{\operatorname{Hom}}\left(Z / W, C_{\delta}\right)=0$. We see that $\operatorname{Hom}(Y, C)=0$, whence $\operatorname{Hom}(X, C)=0$.

Given a localizing subcategory of finite type $\mathcal{S}$ in $\mathcal{C}$, we denote

$$
O(\mathcal{S})=\left\{E \in \operatorname{Sp} \mathcal{C} \mid t_{\mathcal{S}}(E) \neq 0\right\} .
$$

The next result was obtained by Herzog [12 and Krause 13 for locally coherent Grothendieck categories and by Garkusha-Prest [5] for the category of modules $\operatorname{Mod} R$ over a commutative ring $R$.

Theorem 11. Suppose $\mathcal{C}$ is a locally finitely presented Grothendieck category. The collection

$$
\{O(\mathcal{S}) \mid \mathcal{S} \subset \mathcal{C} \text { is a localizing subcategory of finite type }\}
$$

of subsets of $\mathrm{SpC}$ satisfies the axioms for the open sets of a topology on the injective spectrum $\mathrm{Sp} \mathcal{C}$. This topological space will be denoted by $\mathrm{Sp}_{\mathrm{fl}} \mathcal{C}$, and this topology will be referred to as the fl-topology ("fl" for finite localizations). Moreover, the map

$$
\mathcal{S} \longmapsto O(\mathcal{S})
$$

is an inclusion-preserving bijection between the localizing subcategories $\mathcal{S}$ of finite type in $\mathcal{C}$ and the open subsets of $\mathrm{Sp}_{\mathrm{fl}} \mathcal{C}$.

Proof. First, note that $O(\mathcal{S})=(\mathcal{S}), O(0)=\varnothing$, and $O(\mathcal{C})=\operatorname{Sp} \mathcal{C}$. We have $O\left(\mathcal{S}_{1}\right) \cap$ $O\left(\mathcal{S}_{2}\right)=\left(\mathcal{S}_{1} \cap \mathcal{S}_{2}\right)$ by Proposition 4. We claim that $\mathcal{S}_{1} \cap \mathcal{S}_{2}$ is of finite type, whence $O\left(\mathcal{S}_{1}\right) \cap O\left(\mathcal{S}_{2}\right)=O\left(\mathcal{S}_{1} \cap \mathcal{S}_{2}\right)$. Indeed, consider a morphism $f: X \rightarrow S$ from a finitely presented object $X$ to an object $S \in \mathcal{S}_{1} \cap \mathcal{S}_{2}$. From [17, 5.8] it follows that there are finitely generated subobjects $X_{1}, X_{2} \subseteq \operatorname{Ker} f$ such that $X / X_{i} \in \mathcal{S}_{i}, i=0,1$. Then $X_{1}+X_{2}$ is a finitely generated subobject of $\operatorname{Ker} f$ and $X /\left(X_{1}+X_{2}\right) \in \mathcal{S}_{1} \cap \mathcal{S}_{2}$. By [17, 5.8], $\mathcal{S}_{1} \cap \mathcal{S}_{2}$ is of finite type.

By Lemma 10, $\sqrt{ } \bigcup_{i \in I} \mathcal{S}_{i}$ is of finite type if so is each $\mathcal{S}_{i}$. Proposition 4 implies that $\bigcup_{i \in I} O\left(\mathcal{S}_{i}\right)=O\left(\bigcup_{i \in I} \mathcal{S}_{i}\right)=O\left(\sqrt{ } \bigcup_{i \in I} \mathcal{S}_{i}\right)$, and the map (3.1) is bijective.

Let $L_{\mathrm{loc}}(\mathcal{C})$ denote the lattice of localizing subcategories of $\mathcal{C}$, where, by definition,

$$
\mathcal{S} \wedge \mathcal{Q}=\mathcal{S} \cap \mathcal{Q}, \quad \mathcal{S} \vee \mathcal{Q}=\sqrt{ }(\mathcal{S} \cup \mathcal{Q})
$$

for any $\mathcal{S}, \mathcal{Q} \in L_{\text {loc }}(\mathcal{C})$. The proof of Theorem 11 shows that the subset of localizing subcategories of finite type in $L_{\mathrm{loc}}(\mathcal{C})$ is a sublattice. We shall denote it by $L_{\mathrm{f} . l o c}(\mathcal{C})$.

Remark. If $\mathcal{C}$ is a locally coherent Grothendieck category, the topological space $\mathrm{Sp}_{\mathrm{ff}} \mathcal{C}$ is also called in the literature the Ziegler spectrum of $\mathcal{C}$. It arose in Ziegler's paper [19] on the model theory of modules. In accordance with the original Ziegler definition, the points of the Ziegler spectrum of a ring $R$ are the isomorphism classes of indecomposable pure injective right $R$-modules. These can be identified with $\mathrm{Sp}(R \bmod , \mathrm{Ab})$, where $(R \bmod , \mathrm{Ab})$ is the locally coherent Grothendieck category consisting of additive covariant functors defined on the category of finitely presented left modules $R \bmod$ and with values in the category of Abelian groups Ab. Closed subsets correspond to complete theories of 
modules. Later, Herzog [12] and Krause [13] defined the Ziegler topology for arbitrary locally coherent Grothendieck categories.

Proposition 12. For any localizing subcategory of strictly finite type $\mathcal{S}$ in a locally finitely presented Grothendieck category $\mathcal{C}$, the topology on $\operatorname{Sp}_{\mathrm{fl}}(\mathcal{C} / \mathcal{S})$ coincides with the subspace topology induced by $\mathrm{Sp}_{\mathrm{fl}} \mathcal{C}$.

Proof. By [17, 5.9], $\mathcal{C} / \mathcal{S}$ is a locally finitely presented Grothendieck category, so that the fl-topology on $\operatorname{Sp}(\mathcal{C} / \mathcal{S})$ makes sense. Let $O(\mathcal{P})$ be an open subset of $\operatorname{Sp}_{\mathrm{fl}}(\mathcal{C} / \mathcal{S})$ with $\mathcal{P}$ a localizing subcategory of finite type in $\mathcal{C} / \mathcal{S}$. There is a unique localizing subcategory $\mathcal{T}$ of $\mathcal{C}$ such that $(\mathcal{C} / \mathcal{S}) / \mathcal{P} \cong \mathcal{C} / \mathcal{T}$. We claim that $\mathcal{T}$ is of finite type.

Obviously, it suffices to verify that $X=\sum_{\mathcal{C}} X_{\alpha}$ is a $\mathcal{T}$-closed object whenever each $X_{\alpha}$ is $\mathcal{T}$-closed. Since $\mathcal{S}$ and $\mathcal{P}$ are of finite type in $\mathcal{C}$ and $\mathcal{C} / \mathcal{S}$, respectively, $X$ is both $\mathcal{S}$-closed and $\mathcal{P}$-closed in $\mathcal{C}$ and in $\mathcal{C} / \mathcal{S}$, respectively. It follows that $X$ is $\mathcal{T}$-closed. Therefore, $\mathcal{T}$ is of finite type and $O(\mathcal{P})=\operatorname{Sp}_{\mathrm{fl}}(\mathcal{C} / \mathcal{S}) \cap O(\mathcal{T})$.

Now, let $\mathcal{Q}$ be a localizing subcategory of finite type in $\mathcal{C}$. We want to show that $\mathrm{Sp}_{\mathrm{fl}}(\mathcal{C} / \mathcal{S}) \cap O(\mathcal{Q})$ is open in $\operatorname{Sp}_{\mathrm{fl}}(\mathcal{C} / \mathcal{S})$. Let $\hat{\mathcal{Q}}=\left\{X_{\mathcal{S}} \mid X \in \mathcal{Q}\right\}$. Then $\hat{\mathcal{Q}}$ is closed under direct sums, subobjects, and quotient objects in $\mathcal{C} / \mathcal{S}$ (see the proof of Lemma 5 ) and $O(\widehat{\mathcal{Q}})=O(\sqrt{ } \hat{\mathcal{Q}})=\mathrm{Sp}_{\mathrm{fl}}(\mathcal{C} / \mathcal{S}) \cap O(\mathcal{Q})$. We must show that $\sqrt{ } \widehat{\mathcal{Q}}$ is of finite type in $\mathcal{C} / \mathcal{S}$

If we check that every direct limit $C=\lim _{\mathcal{C} / \mathcal{S}} C_{\delta}$ of $\sqrt{ } \widehat{\mathcal{Q}}$-closed objects $C_{\delta}$ has no $\sqrt{ } \hat{\mathcal{Q}}$-torsion, then from [17, 5.8] it will follow that $\sqrt{ } \widehat{\mathcal{Q}}$ is of finite type. Obviously, each $C_{\delta}$ is $\mathcal{Q}$-closed.

By Proposition 2, it suffices to check that $\operatorname{Hom}_{\mathcal{C}}(X, C)=0$ for any object $X \in \hat{\mathcal{Q}}$. Since $\mathcal{S}$ is of strictly finite type, we have $C \cong \lim _{\mathcal{C}} C_{\delta}$. Since each $C_{\delta}$ is $\mathcal{Q}$-closed, $\lim _{\mathcal{C}} C_{\delta}$ has no $\mathcal{Q}$-torsion by [17, 5.8] and the fact that $\mathcal{Q}$ is of finite type. There is an object $Y \in \mathcal{Q}$ such that $Y_{\mathcal{S}}=X$. Then $\operatorname{Hom}_{\mathcal{C} / \mathcal{S}}(X, C) \cong \operatorname{Hom}_{\mathcal{C}}\left(Y, \lim _{\mathcal{C}} C_{\delta}\right)=0$, as required.

\section{$\S 4$. The topological space $\mathrm{Sp}_{\mathrm{fl}, \otimes}(\mathrm{X})$}

In the preceding section we studied some general properties of finite localizations in locally finitely presented Grothendieck categories and their relationship with the topological space $\mathrm{Sp}_{\mathrm{ff}} \mathcal{C}$. In this section we introduce and study the topological space $\mathrm{Sp}_{\mathrm{fl}, \otimes}(X)$, which is of particular importance in practice. Unless otherwise specified, $X$ is assumed to be a quasicompact and quasiseparated scheme.

Given a quasicompact open subset $U \subset X$, we denote $\mathcal{S}_{U}=\left\{\mathcal{F} \in \mathrm{Q} \operatorname{coh}(X)|\mathcal{F}|_{U}=\right.$ $0\}$. Using [17, 5.9] and the fact that $\left.\mathcal{F}\right|_{U} \in \mathrm{fp}(\mathrm{Q} \operatorname{coh}(U))$ whenever $\mathcal{F} \in \mathrm{fp}(\mathrm{Q} \operatorname{coh}(X))$, we see that $\mathcal{S}_{U}$ is of strictly finite type. Below we shall need the following statement.

Lemma 13. Let $X$ be a quasicompact and quasiseparated scheme, and let $U, V$ be quasicompact open subsets. Then

$$
\mathcal{S}_{U \cap V}=\sqrt{ }\left(\mathcal{S}_{U} \cup \mathcal{S}_{V}\right) .
$$

Proof. Clearly, $\mathcal{S}_{U \cap V}$ contains both $\mathcal{S}_{U}$ and $\mathcal{S}_{V}$, whence $\mathcal{S}_{U \cap V} \supset \sqrt{ }\left(\mathcal{S}_{U} \cup \mathcal{S}_{V}\right)$. Let $\mathcal{F} \in \mathcal{S}_{U \cap V}$, and let $j: U \rightarrow X$ be the canonical inclusion. Then $j_{*} j^{*}(\mathcal{F}) \in \mathcal{S}_{V}$. We have the following exact sequence:

$$
0 \rightarrow t_{\mathcal{S}_{U}}(\mathcal{F}) \rightarrow \mathcal{F} \stackrel{\lambda_{\mathcal{F}}}{\longrightarrow} j_{*} j^{*}(\mathcal{F}) .
$$

Since $t_{\mathcal{S}_{U}}(\mathcal{F}) \in \mathcal{S}_{U}$ and $\operatorname{Im}\left(\lambda_{\mathcal{F}}\right) \in \mathcal{S}_{V}$, we see that $\mathcal{F} \in \sqrt{ }\left(\mathcal{S}_{U} \cup \mathcal{S}_{V}\right)$.

We denote by $\operatorname{Sp}_{\mathrm{fl}}(X)$ the topological space $\mathrm{Sp}_{\mathrm{fl}}(\mathrm{Q} \operatorname{coh}(X))$. 
Corollary 14. Let $X$ be a quasicompact and quasiseparated scheme and let $X=U \cup V$, where $U, V$ are quasicompact open subsets. Then

$$
\begin{aligned}
\mathrm{Sp}(X) & =\mathrm{Sp}(U) \cup \mathrm{Sp}(V), & & \mathrm{Sp}(U \cap V)=\mathrm{Sp}(U) \cap \mathrm{Sp}(V), \\
\mathrm{Sp}_{\mathrm{fl}}(X) & =\mathrm{Sp}_{\mathrm{fl}}(U) \cup \mathrm{Sp}_{\mathrm{fl}}(V), & & \operatorname{Sp}_{\mathrm{fl}}(U \cap V)=\operatorname{Sp}_{\mathrm{fl}}(U) \cap \mathrm{Sp}_{\mathrm{ff}}(V) .
\end{aligned}
$$

Proof. This follows from the fact that $\mathcal{S}_{U} \cap \mathcal{S}_{V}=0$, Propositions 1, 4, 6, 12, Theorem 11, and Lemma 13

Let $L_{\mathrm{loc}}(X)$ (respectively, $\left.L_{\mathrm{f} . \mathrm{loc}}(X)\right)$ denote the lattice $L_{\mathrm{loc}}(\mathrm{Q} \operatorname{coh}(X))$ (respectively, $\left.L_{\text {f.loc }}(\mathrm{Q} \operatorname{coh}(X))\right)$. By Proposition 4 and Theorem 11, the map $L_{\mathrm{loc}}(X) \rightarrow L_{\text {open }}(\operatorname{Sp}(X))$ (respectively, $\left.L_{\mathrm{f} . \text { loc }}(X) \rightarrow L_{\mathrm{open}}\left(\operatorname{Sp}_{\mathrm{fl}}(X)\right)\right)$ is a lattice isomorphism. Suppose $U \subset X$ is a quasicompact open subset. Then the map

$$
\alpha_{X, U}: L_{\mathrm{loc}}(X) \rightarrow L_{\mathrm{loc}}(U), \quad \mathcal{S} \mapsto \sqrt{ }\left(\left.\hat{\mathcal{S}}\right|_{U}\right),
$$

where $\left.\hat{\mathcal{S}}\right|_{U}=\left\{\left.\mathcal{F}\right|_{U}=\mathcal{F}_{\mathcal{S}_{U}} \mid \mathcal{F} \in \mathcal{S}\right\}$, is a lattice map. If $V$ is another quasicompact subset of $X$ such that $X=U \cup V$, then, obviously,

$$
\alpha_{X, U \cap V}=\alpha_{U, U \cap V} \circ \alpha_{X, U}=\alpha_{V, U \cap V} \circ \alpha_{X, V} .
$$

By the proof of Proposition 12, $\alpha_{X, U}(\mathcal{S}) \in L_{\text {f.loc }}(U)$ for every $\mathcal{S} \in L_{\text {f.loc }}(U)$. Thus, we have a map

$$
\alpha_{X, U}: L_{\mathrm{f} . \text { loc }}(X) \rightarrow L_{\mathrm{f} . \mathrm{loc}}(U) .
$$

The notion of a pullback for lattices satisfying the obvious universal property is defined easily.

Lemma 15. The commutative squares of lattices

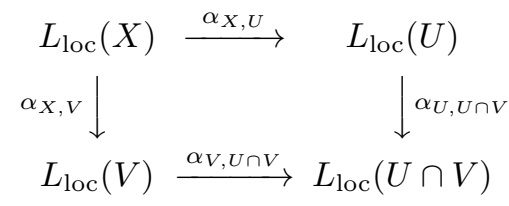

and

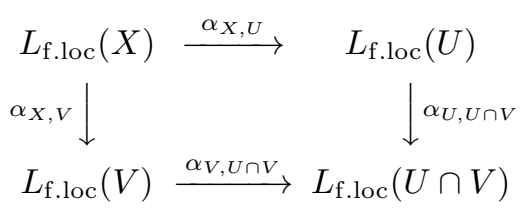

are pullback.

Proof. It suffices to observe that the commutative squares in the lemma are isomorphic to the corresponding pullback squares of lattices of open sets

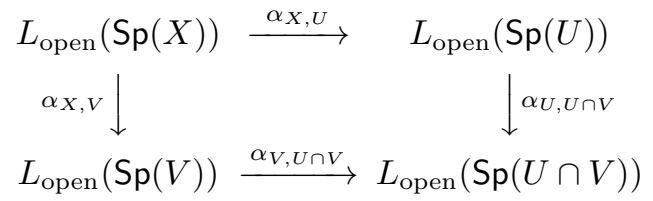

and

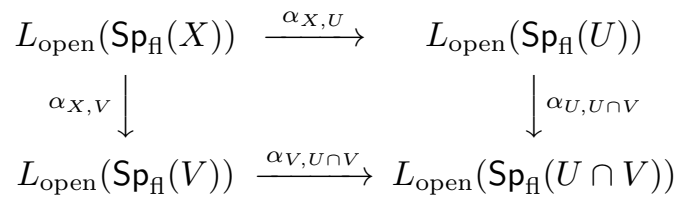

(see Proposition 4, Theorem [11, and Corollary 14). 
Recall that $\mathrm{Q} \operatorname{coh}(X)$ is monoidal, and the tensor product $\otimes_{X}$ is right exact and preserves direct limits (see [20, §II.2]).

Definition. A localizing subcategory $\mathcal{S}$ of $\mathrm{Q} \operatorname{coh}(X)$ is said to be tensor if $\mathcal{F} \otimes_{X} \mathcal{G} \in \mathcal{S}$ for every $\mathcal{F} \in \mathcal{S}$ and $\mathcal{G} \in \mathrm{Q} \operatorname{coh}(X)$.

Lemma 16. A localizing subcategory of finite type $\mathcal{S} \subset \mathrm{Q} \operatorname{coh}(X)$ is tensor if and only if $\mathcal{F} \otimes_{X} \mathcal{G} \in \mathcal{S}$ for every $\mathcal{F} \in \mathcal{S} \cap \mathrm{fp}(\mathrm{Qcoh}(X))$ and $\mathcal{G} \in \mathrm{fp}(\mathrm{Qcoh}(X))$.

Proof. It suffices to observe that every $\mathcal{F} \in \mathcal{S}$ is a quotient object of the direct sum of objects from $\mathcal{S} \cap \mathrm{fp}(\mathrm{Q} \operatorname{coh}(X))$ and that every object $\mathcal{G} \in \mathrm{Q} \operatorname{coh}(X)$ is a direct limit of finitely presented objects.

Lemma 17. Let $\mathcal{X} \subset \mathrm{Q} \operatorname{coh}(X)$ be a tensor subcategory closed under direct sums, subobjects, and quotient objects. Then $\sqrt{ } \mathcal{X}$ is a tensor localizing subcategory.

Proof. By Proposition 2] an object $\mathcal{F}$ belongs to $\sqrt{ } \mathcal{X}$ if and only if there is a filtration

$$
\mathcal{F}_{0} \subset \mathcal{F}_{1} \subset \cdots \subset \mathcal{F}_{\beta} \subset \cdots
$$

such that $\mathcal{F}=\bigcup_{\beta} \mathcal{F}_{\beta}, \mathcal{F}_{\gamma}=\bigcup_{\beta<\gamma} \mathcal{F}_{\beta}$ if $\gamma$ is a limit ordinal, and $\mathcal{F}_{0}, \mathcal{F}_{\beta+1} / \mathcal{F}_{\beta} \in\left\langle\mathcal{X}^{\oplus}\right\rangle=$ $\mathcal{X}$.

We have $\mathcal{F}_{0} \otimes_{X} \mathcal{G} \in \mathcal{X}$ for any $\mathcal{G} \in \mathrm{Q} \operatorname{coh}(X)$. Suppose $\beta=\alpha+1$ and $\mathcal{F}_{\alpha} \otimes_{X} \mathcal{G} \in \sqrt{ } \mathcal{X}$. There is an exact sequence

$$
\mathcal{F}_{\alpha} \otimes_{X} \mathcal{G} \stackrel{f}{\longrightarrow} \mathcal{F}_{\beta} \otimes_{X} \mathcal{G} \rightarrow\left(\mathcal{F}_{\beta} / \mathcal{F}_{\alpha}\right) \otimes_{X} \mathcal{G} \rightarrow 0 .
$$

Since $\left(\mathcal{F}_{\beta} / \mathcal{F}_{\alpha}\right) \otimes_{X} \mathcal{G} \in \mathcal{X}$ and $\operatorname{Im} f \in \sqrt{ } \mathcal{X}$, we see that $\mathcal{F}_{\beta} \otimes_{X} \mathcal{G} \in \sqrt{ } \mathcal{X}$. If $\gamma$ is a limit ordinal and $\mathcal{F}_{\beta} \otimes_{X} \mathcal{G} \in \sqrt{ } \mathcal{X}$ for all $\beta<\gamma$, then $\mathcal{F}_{\gamma} \otimes_{X} \mathcal{G}=\lim _{\beta<\gamma}\left(\mathcal{F}_{\beta} \otimes_{X} \mathcal{G}\right) \in \sqrt{ } \mathcal{X}$. Therefore, $\sqrt{ } \mathcal{X}$ is tensor.

The next statement is of great importance in this paper.

Reduction principle. Let $\mathfrak{S}$ be the class of quasicompact and quasiseparated schemes, and let $P$ be a property satisfied by some schemes in $\mathfrak{S}$. Moreover, assume the following.

(1) $P$ is true for affine schemes.

(2) If $X \in \mathfrak{S}, X=U \cup V$, where $U, V$ are quasicompact open subsets of $X$, and $P$ holds for $U, V$, and $U \cap V$, then it holds for $X$.

Then $P$ holds for all schemes from $\mathfrak{S}$.

Proof. See the proof in [21, 3.9.2.4] and [22, 3.3.1].

Lemma 18. The join $\mathcal{T}=\sqrt{ }(\mathcal{S} \cup \mathcal{Q})$ of two tensor localizing subcategories $\mathcal{S}, \mathcal{Q} \subset$ $\mathrm{Q} \operatorname{coh}(X)$ is tensor.

Proof. We use the reduction principle to demonstrate the lemma. It is true for affine schemes, because every localizing subcategory is tensor in this case. Suppose $X=U \cup V$, where $U, V$ are quasicompact open subsets of $X$, and the assertion is true for $U, V$, and $U \cap V$. We must show that it is true for $X$ itself.

We have the following relation:

$$
\alpha_{X, U}(\mathcal{T})=\left(\sqrt{ }\left(\left.\widehat{\mathcal{S}}\right|_{U}\right)\right) \vee\left(\sqrt{ }\left(\left.\widehat{\mathcal{Q}}\right|_{U}\right)\right) .
$$

For any $\mathcal{F}, \mathcal{G} \in \mathrm{Q} \operatorname{coh}(X)$, there is a canonical isomorphism (see [20, II.2.3.5])

$$
\left.\left(\left.\mathcal{F}\right|_{U}\right) \otimes_{U}\left(\left.\mathcal{G}\right|_{U}\right) \cong\left(\mathcal{F} \otimes_{X} \mathcal{G}\right)\right|_{U}
$$

It follows that both $\left.\hat{\mathcal{S}}\right|_{U}$ and $\left.\hat{\mathcal{Q}}\right|_{U}$ are closed under tensor products. By Lemma 17 , both $\left.\sqrt{ } \widehat{\mathcal{S}}\right|_{U}$ and $\left.\sqrt{ } \hat{\mathcal{Q}}\right|_{U}$ are tensor. By assumption, the join of two tensor localizing subcategories in $\operatorname{Qcoh}(U)$ is tensor, whence $\alpha_{X, U}(\mathcal{T})$ is tensor. For the same reasons, 
$\alpha_{X, V}(\mathcal{T})$ is tensor. Obviously, $\mathcal{T}$ is tensor whenever so are $\alpha_{X, U}(\mathcal{T})$ and $\alpha_{X, V}(\mathcal{T})$. Therefore, $\mathcal{T}$ is also tensor, and now our assertion follows from the reduction principle.

Given a tensor localizing subcategory $\mathcal{S}$ of finite type in $\mathrm{Qcoh}(X)$, we denote

$$
\mathcal{O}(\mathcal{S})=\left\{\mathcal{E} \in \operatorname{Sp}(X) \mid t_{\mathcal{S}}(\mathcal{E}) \neq 0\right\} .
$$

Theorem 19. The collection

$$
\{\mathcal{O}(\mathcal{S}) \mid \mathcal{S} \subset \mathrm{Qcoh}(X) \text { is a tensor localizing subcategory of finite type }\}
$$

of subsets of the injective spectrum $\mathrm{Sp}(X)$ satisfies the axioms for the open sets of a topology on $\mathrm{Sp}(X)$. This topological space will be denoted by $\mathrm{Sp}_{\mathrm{fl}, \otimes}(X)$, and this topology will be referred to as the tensor fl-topology. Moreover, the map

$$
\mathcal{S} \longmapsto \mathcal{O}(\mathcal{S})
$$

is an inclusion-preserving bijection between the tensor localizing subcategories $\mathcal{S}$ of finite type in $\mathrm{Q} \operatorname{coh}(X)$ and the open subsets of $\mathrm{Sp}_{\mathrm{fl}, \otimes}(X)$.

Proof. Obviously, the intersection $\mathcal{S}_{1} \cap \mathcal{S}_{2}$ of two tensor localizing subcategories of finite type is a tensor localizing subcategory of finite type; hence, $\mathcal{O}\left(\mathcal{S}_{1} \cap \mathcal{S}_{2}\right)=\mathcal{O}\left(\mathcal{S}_{1}\right) \cap \mathcal{O}\left(\mathcal{S}_{2}\right)$ by Theorem 11.

Now we show that the join $\mathcal{T}=\sqrt{ }\left(\bigcup_{i \in I} \mathcal{S}_{i}\right)$ of tensor localizing subcategories of finite type $\mathcal{S}_{i}$ is tensor. By induction and Lemma $18, \mathcal{T}$ is tensor whenever $I$ is finite. Now, without loss of generality, we may assume that $I$ is a directed index set and $\mathcal{S}_{i} \subset \mathcal{S}_{j}$ for any $i \leq j$. By the proof of Lemma 10, $\mathcal{T}=\sqrt{ } \mathcal{X}$, where $\mathcal{X}$ is the full subcategory in $\mathrm{Q} \operatorname{coh}(X)$ of objects that can be presented as directed sums $\sum F_{\alpha}$ with each $F_{\alpha}$ in $\bigcup_{i \in I} \mathcal{S}_{i}$. Then $\mathcal{X}$ is closed under direct sums, subobjects, and quotient objects. It is also closed under tensor product, because $\otimes_{X}$ commutes with direct limits. By Lemma 17. $\mathcal{T}$ is tensor, and by Lemma 10, $\mathcal{T}$ is of finite type.

Theorem 11 implies that $\mathcal{O}\left(\sqrt{ }\left(\bigcup_{i \in I} \mathcal{S}_{i}\right)\right)=\bigcup_{i \in I} \mathcal{O}\left(\mathcal{S}_{i}\right)$ and the map (4.1) is a bijection.

We denote by $L_{\mathrm{f} . l o c} \otimes(X)$ the lattice of tensor localizing subcategories of finite type in $\mathrm{Qcoh}(X)$.

Corollary 20. The commutative square of lattices

is pullback.

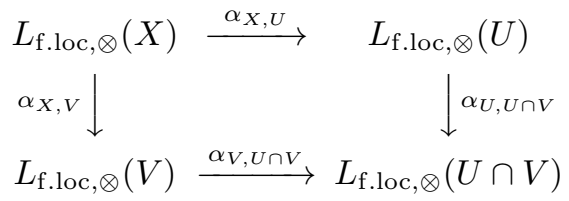

Proof. The proof is similar to that of Lemma 15.

\section{$\S 5$. THE ClASSIFICATION THEOREM}

We recall (see [23]) that a topological space is spectral if it is $T_{0}$ and quasicompact, the quasicompact open subsets are closed under finite intersections and form an open basis, and every nonempty irreducible closed subset has a generic point. Given a spectral topological space $X$, Hochster [23] endowed the underlying set with a new, "dual", topology, denoted $X^{*}$, by taking as open sets those of the form $Y=\bigcup_{i \in \Omega} Y_{i}$, where $Y_{i}$ has quasicompact open complement $X \backslash Y_{i}$ for all $i \in \Omega$. Then $X^{*}$ is spectral and $\left(X^{*}\right)^{*}=X($ see $[23$, Proposition 8]). 
As an example, the underlying topological space of a quasicompact, quasiseparated scheme $X$ is spectral. In this section we show that the tensor localizing subcategories of finite type in $\mathrm{Q} c o h(X)$ are in one-to-one correspondence with the open subsets of $X^{*}$. Unless otherwise specified, $X$ is assumed to be a quasicompact, quasiseparated scheme.

Given a quasicompact open subset $D \subset X$, we denote $\mathcal{S}_{D}=\{\mathcal{F} \in \operatorname{Qcoh}(X) \mid$ $\left.\left.\mathcal{F}\right|_{D}=0\right\}$.

Proposition 21. For any open subset $O=\bigcup_{I} O_{i} \subset X^{*}$, where each $D_{i}=X \backslash O_{i}$ is quasicompact and open in $X$, the subcategory $\mathcal{S}=\left\{\mathcal{F} \in \mathrm{Q} \operatorname{coh}(X) \mid \operatorname{supp}_{X}(\mathcal{F}) \subseteq O\right\}$ is a tensor localizing subcategory of finite type, and $\mathcal{S}=\sqrt{ }\left(\bigcup_{I} \mathcal{S}_{D_{i}}\right)$.

Proof. Given a short exact sequence

$$
\mathcal{F}^{\prime} \longmapsto \mathcal{F} \rightarrow \mathcal{F}^{\prime \prime}
$$

in $\operatorname{Qcoh}(X)$, we have $\operatorname{supp}_{X}(\mathcal{F})=\operatorname{supp}_{X}\left(\mathcal{F}^{\prime}\right) \cup \operatorname{supp}_{X}\left(\mathcal{F}^{\prime \prime}\right)$. It follows that $\mathcal{S}$ is a Serre subcategory. It is also closed under direct sums, hence localizing, because $\operatorname{supp}_{X}\left(\bigoplus_{I} \mathcal{F}_{i}\right)$ $=\bigcup_{I} \operatorname{supp}_{X}\left(\mathcal{F}_{i}\right)$.

We use the reduction principle to show that $\mathcal{S}$ is a tensor localizing subcategory of finite type and $\mathcal{S}=\sqrt{ }\left(\bigcup_{I} \mathcal{S}_{D_{i}}\right)$. It is the case for affine schemes (see [5, 2.2]). Suppose $X=U \cup V$, where $U, V$ are quasicompact open subsets of $X$, and the assertion is true for $U, V$, and $U \cap V$. We need to show that it is true for $X$ itself.

For any $\mathcal{F} \in Q \operatorname{coh}(X)$ we have

$$
\operatorname{supp}_{X}(\mathcal{F})=\operatorname{supp}_{U}\left(\left.\mathcal{F}\right|_{U}\right) \cup \operatorname{supp}_{V}\left(\left.\mathcal{F}\right|_{V}\right) .
$$

Clearly, $O \cap U$ is open in $U^{*}$ and $\operatorname{supp}_{U}\left(\left.\mathcal{F}\right|_{U}\right) \subseteq O \cap U$ for any $\mathcal{F} \in \mathcal{S}$. We see that $\left.\widehat{\mathcal{S}}\right|_{U}=\left\{\left.\mathcal{F}\right|_{U}=\mathcal{F}_{\mathcal{S}_{U}} \mid \mathcal{F} \in \mathcal{S}\right\}$ is contained in $\mathcal{S}(U)=\left\{\mathcal{F} \in \operatorname{Qcoh}(U) \mid \operatorname{supp}_{U}(\mathcal{F}) \subseteq\right.$ $O \cap U\}$. By assumption, $\mathcal{S}(U)$ is a tensor localizing subcategory of finite type in $\mathrm{Q} \operatorname{coh}(U)$ and $\mathcal{S}(U)=\sqrt{ }\left(\bigcup_{I} \mathcal{S}_{D_{i} \cap U}\right)$. We have $\mathcal{S}(U) \supset \sqrt{ }\left(\left.\widehat{\mathcal{S}}\right|_{U}\right)$. Similarly, $\mathcal{S}(V) \supset \sqrt{ }\left(\left.\widehat{\mathcal{S}}\right|_{V}\right)$ and $\mathcal{S}(V)=\sqrt{ }\left(\bigcup_{I} \mathcal{S}_{D_{i} \cap V}\right)$.

Since

$$
\begin{aligned}
\alpha_{U, U \cap V}\left(\mathcal{S}_{D_{i} \cap U}\right) & =\alpha_{V, U \cap V}\left(\mathcal{S}_{D_{i} \cap V}\right) \stackrel{\text { Lem.13 }}{=} \mathcal{S}_{D_{i} \cap U \cap V} \\
& \stackrel{\text { Prop. } 1}{=}\left\{\mathcal{F} \in \operatorname{Qcoh}(U \cap V) \mid \operatorname{supp}_{U \cap V}(\mathcal{F}) \subseteq O_{i} \cap U \cap V\right\},
\end{aligned}
$$

it follows that

$$
\begin{aligned}
\alpha_{U, U \cap V}(\mathcal{S}(U)) & =\alpha_{V, U \cap V}(\mathcal{S}(V))=\mathcal{S}(U \cap V) \\
& =\left\{\mathcal{F} \in \operatorname{Q} \operatorname{coh}(U \cap V) \mid \operatorname{supp}_{U \cap V}(\mathcal{F}) \subseteq O \cap U \cap V\right\}=\sqrt{ }\left(\bigcup_{I} \mathcal{S}_{D_{i} \cap U \cap V}\right) .
\end{aligned}
$$

By Corollary 20, there is a unique tensor localizing subcategory of finite type $\mathcal{T} \in$ $L_{\text {f.loc }, \otimes}(X)$ such that $\sqrt{ }\left(\left.\hat{\mathcal{T}}\right|_{U}\right)=\mathcal{S}(U), \sqrt{ }\left(\left.\hat{\mathcal{T}}\right|_{V}\right)=\mathcal{S}(V)$ and $\mathcal{T}=\sqrt{ }\left(\bigcup_{I} \mathcal{S}_{D_{i}}\right)$. By construction, $\operatorname{supp}_{X}(\mathcal{F}) \subseteq O$ for all $\mathcal{F} \in \mathcal{T}$, whence $\mathcal{T} \subseteq \mathcal{S}, \sqrt{ }\left(\left.\hat{\mathcal{T}}\right|_{U}\right) \subseteq \sqrt{ }\left(\left.\hat{\mathcal{S}}\right|_{U}\right)$, $\sqrt{ }\left(\left.\widehat{\mathcal{T}}\right|_{V}\right) \subseteq \sqrt{ }\left(\left.\widehat{\mathcal{S}}\right|_{V}\right)$. Therefore, $\mathcal{S}(V)=\sqrt{ }\left(\left.\widehat{\mathcal{S}}\right|_{V}\right)$ and $\mathcal{S}(V)=\sqrt{ }\left(\left.\widehat{\mathcal{S}}\right|_{V}\right)$. By Corollary 20, we have $\mathcal{S}=\mathcal{T}$.

Let $X=U \cup V$ with $U, V$ open and quasicompact. Then $X^{*}=U^{*} \cup V^{*}$ and both $U^{*}$ and $V^{*}$ are closed subsets of $X^{*}$. Let $Y \in L_{\text {open }}\left(X^{*}\right)$; then $Y=\bigcup_{I} Y_{i}$, where each $D_{i}:=X \backslash Y_{i}$ is an open and quasicompact subset in $X$. Since each $D_{i} \cap U$ is an open and quasicompact subset in $U$, it follows that $Y \cap U=\bigcup_{I}\left(Y_{i} \cap U\right) \in L_{\text {open }}\left(U^{*}\right)$. Then the map

$$
\beta_{X, U}: L_{\text {open }}\left(X^{*}\right) \rightarrow L_{\text {open }}\left(U^{*}\right), \quad Y \mapsto Y \cap U
$$

is a lattice map. The lattice map $\beta_{X, V}: L_{\text {open }}\left(X^{*}\right) \rightarrow L_{\text {open }}\left(V^{*}\right)$ is defined similarly. 
Lemma 22. The square

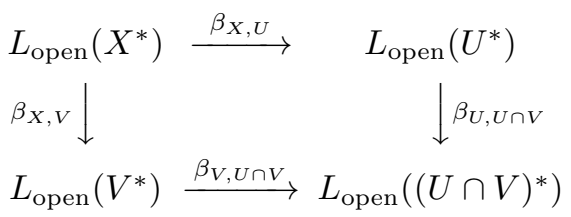

is commutative and pullback.

Proof. It is easily seen that the lattice maps

$$
Y \in L_{\mathrm{open}}\left(X^{*}\right) \mapsto(Y \cap U, Y \cap V) \in L_{\mathrm{open}}\left(U^{*}\right) \prod_{L_{\mathrm{open}}\left((U \cap V)^{*}\right)} L_{\mathrm{open}}\left(V^{*}\right)
$$

and

$$
\left(Y_{1}, Y_{2}\right) \in L_{\mathrm{open}}\left(U^{*}\right) \prod_{L_{\mathrm{open}}\left((U \cap V)^{*}\right)} L_{\mathrm{open}}\left(V^{*}\right) \mapsto Y_{1} \cup Y_{2} \in L_{\mathrm{open}}\left(X^{*}\right)
$$

are mutually inverse.

Lemma 23. Given a subcategory $\mathcal{X}$ in $\mathrm{Q} \operatorname{coh}(X)$, we have

$$
\bigcup_{\mathcal{F} \in \mathcal{X}} \operatorname{supp}_{X}(\mathcal{F})=\bigcup_{\mathcal{F} \in \sqrt{ } \mathcal{X}} \operatorname{supp}_{X}(\mathcal{F})
$$

Proof. Since $\operatorname{supp}_{X}\left(\bigoplus_{I} \mathcal{F}_{i}\right)=\bigcup_{I} \operatorname{supp}_{X}\left(\mathcal{F}_{i}\right)$ and $\operatorname{supp}_{X}(\mathcal{F})=\operatorname{supp}_{X}\left(\mathcal{F}^{\prime}\right) \cup \operatorname{supp}_{X}\left(\mathcal{F}^{\prime \prime}\right)$ for any short exact sequence $\mathcal{F}^{\prime} \longleftrightarrow \mathcal{F} \rightarrow \mathcal{F}^{\prime \prime}$ in $\mathrm{Q} \operatorname{coh}(X)$, we may assume that $\mathcal{X}$ is closed under subobjects, quotient objects, and direct sums, i.e., $\mathcal{X}=\left\langle\mathcal{X}^{\oplus}\right\rangle$. If $\mathcal{F}=\sum_{I} F_{i}$, we also have $\operatorname{supp}_{X}(\mathcal{F}) \subseteq \bigcup_{I} \operatorname{supp}_{X}\left(\mathcal{F}_{i}\right)$. Now our assertion follows from Proposition 2 ,

Lemma 24. For any tensor localizing subcategory of finite type $\mathcal{S} \in L_{\mathrm{f} . l \mathrm{loc}, \otimes}(X)$, the set

$$
Y=\bigcup_{\mathcal{F} \in \mathcal{S}} \operatorname{supp}_{X}(\mathcal{F})
$$

is open in $X^{*}$.

Proof. We use the reduction principle to show that $Y \in L_{\text {open }}\left(X^{*}\right)$. This is the case for the affine schemes (see [5, 2.2]). Suppose $X=U \cup V$, where $U, V$ are quasicompact open subsets of $X$, and the assertion is true for $U, V$, and $U \cap V$. We need to show that it is true for $X$ itself.

By Corollary 20,

$$
\alpha_{X, U}(\mathcal{S})=\sqrt{ }\left(\left.\hat{\mathcal{S}}\right|_{U}\right) \in L_{\text {f.loc }, \otimes}(U) \quad \text { and } \quad \alpha_{X, V}(\mathcal{S})=\sqrt{ }\left(\left.\hat{\mathcal{S}}\right|_{V}\right) \in L_{\text {f.loc }, \otimes}(V) .
$$

By assumption,

$$
Y_{1}=\bigcup_{\left.\mathcal{F} \in \sqrt{ } \hat{\mathcal{S}}\right|_{U}} \operatorname{supp}_{U}(\mathcal{F}) \in L_{\text {open }}\left(U^{*}\right)
$$

and

By Lemma 23 ,

$$
Y_{2}=\bigcup_{\left.\mathcal{F} \in \sqrt{\mathcal{S}}\right|_{V}} \operatorname{supp}_{V}(\mathcal{F}) \in L_{\text {open }}\left(V^{*}\right)
$$

$$
Y_{1}=\bigcup_{\left.\mathcal{F} \in \hat{\mathcal{S}}\right|_{U}} \operatorname{supp}_{U}(\mathcal{F})=\bigcup_{\mathcal{F} \in \mathcal{S}} \operatorname{supp}_{U}\left(\left.\mathcal{F}\right|_{U}\right)
$$

and

$$
Y_{2}=\bigcup_{\left.\mathcal{F} \in \hat{\mathcal{S}}\right|_{V}} \operatorname{supp}_{V}(\mathcal{F})=\bigcup_{\mathcal{F} \in \mathcal{S}} \operatorname{supp}_{V}\left(\left.\mathcal{F}\right|_{V}\right)
$$


For every $\mathcal{F} \in \operatorname{Qcoh}(X)$ we have $\operatorname{supp}_{X}(\mathcal{F})=\operatorname{supp}_{U}\left(\left.\mathcal{F}\right|_{U}\right) \cup \operatorname{supp}_{V}\left(\left.\mathcal{F}\right|_{V}\right)$. Therefore, $Y_{1}=Y \cap U$ and $Y_{2}=Y \cap V$. By Lemma 22, $Y=Y_{1} \cup Y_{2} \in L_{\text {open }}\left(X^{*}\right)$.

Now we are in a position to prove the main result of the paper.

Theorem 25 (Classification; see Garkusha-Prest [5] for affine schemes). Let $X$ be a quasicompact, quasiseparated scheme. Then the maps

$$
Y \stackrel{\varphi_{X}}{\longmapsto} \mathcal{S}(Y)=\left\{\mathcal{F} \in \mathrm{Q} \operatorname{coh}(X) \mid \operatorname{supp}_{X}(\mathcal{F}) \subseteq Y\right\}
$$

and

$$
\mathcal{S} \stackrel{\psi_{X}}{\longrightarrow} Y(\mathcal{S})=\bigcup_{\mathcal{F} \in \mathcal{S}} \operatorname{supp}_{X}(\mathcal{F})
$$

induce bijections between

(1) the set of all subsets of the form $Y=\bigcup_{i \in \Omega} Y_{i}$ such that $Y_{i}$ has a quasicompact open complement $X \backslash Y_{i}$ for all $i \in \Omega$; i.e., the set of all open subsets of $X^{*}$, and

(2) the set of all tensor localizing subcategories of finite type in $\mathrm{Qcoh}(X)$.

Moreover, $\mathcal{S}(Y)=\sqrt{ }\left(\bigcup_{i \in I} \mathcal{S}\left(Y_{i}\right)\right)=\sqrt{ }\left(\bigcup_{i \in I} \mathcal{S}_{D_{i}}\right)$, where $D_{i}=X \backslash Y_{i}$ and $\mathcal{S}_{D_{i}}=$ $\left\{\mathcal{F} \in \operatorname{Qcoh}(X)|\mathcal{F}|_{D_{i}}=0\right\}$.

Proof. By Proposition 21 and Lemma 24, $\varphi_{X}(Y) \in L_{\text {f.loc }, \otimes}(X)$ and $\psi_{X}(\mathcal{S}) \in L_{\text {open }}\left(X^{*}\right)$. We have lattice maps

$$
\varphi_{X}: L_{\mathrm{open}}\left(X^{*}\right) \rightarrow L_{\mathrm{f} . \text { loc }, \otimes}(X), \quad \psi_{X}: L_{\mathrm{f} . \text { loc }, \otimes}(X) \rightarrow L_{\mathrm{open}}\left(X^{*}\right) .
$$

We use the reduction principle to show that $\varphi_{X} \psi_{X}=1$ and $\psi_{X} \varphi_{X}=1$. This is the case for the affine schemes (see [5, 2.2]). Suppose $X=U \cup V$, where $U, V$ are quasicompact open subsets of $X$, and the assertion is true for $U, V$, and $U \cap V$. We need to show that it is true for $X$ itself.

Consider the following commutative diagram of lattices:

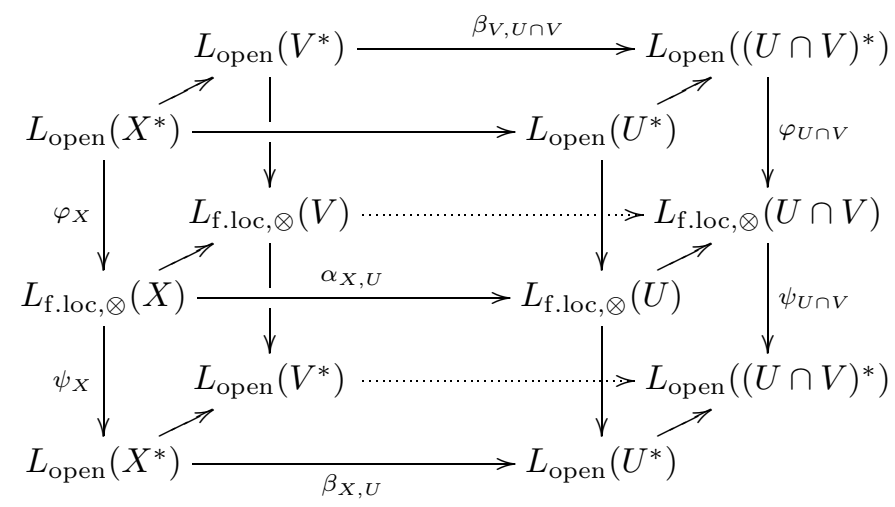

By assumption, all vertical arrows except $\varphi_{X}, \psi_{X}$ are bijections. Namely, the maps $\varphi_{U}, \psi_{U}$ (respectively, $\varphi_{V}, \psi_{V}$ and $\varphi_{U \cap V}, \psi_{U \cap V}$ ) are mutual inverses. Since each horizontal square is pullback (see Corollary 20 and Lemma 221), it follows that $\varphi_{X}, \psi_{X}$ are mutual inverses.

The fact that $\mathcal{S}(Y)=\sqrt{ }\left(\bigcup_{i \in I} \mathcal{S}\left(Y_{i}\right)\right)=\sqrt{ }\left(\left.\bigcup_{i \in I} \mathcal{S}\right|_{D_{i}}\right)$ is a consequence of Propositions 1 and 21. The theorem is proved.

We denote by $\mathcal{D}_{\text {per }}(X)$ the derived category of perfect complexes, i.e., the homotopy category of the complexes of sheaves of $\mathcal{O}_{X}$-modules that are locally quasi-isomorphic to a bounded complex of free $\mathcal{O}_{X}$-modules of finite type. We say that a thick triangulated subcategory $\mathcal{A} \subset \mathcal{D}_{\text {per }}(X)$ is a tensor subcategory if for each object $E$ in $\mathcal{D}_{\text {per }}(X)$ and each $A$ in $\mathcal{A}$, the derived tensor product $E \otimes_{X}^{L} A$ is also in $\mathcal{A}$. 
Let $E$ be a complex of sheaves of $\mathcal{O}_{X}$-modules. The cohomological support of $E$ is the subspace supph $_{X}(E) \subseteq X$ of all points $x \in X$ at which the stalk complex of $\mathcal{O}_{X, x}$-modules $E_{x}$ is not acyclic. Thus supph ${ }_{X}(E)=\bigcup_{n \in \mathbb{Z}} \operatorname{supp}_{X}\left(H_{n}(E)\right)$ is the union of the supports in the classical sense of the cohomology sheaves of $E$.

We shall write $L_{\text {thick }}\left(\mathcal{D}_{\text {per }}(X)\right)$ to denote the lattice of all thick subcategories of $\mathcal{D}_{\text {per }}(X)$.

Theorem 26 (Thomason 6]). Let $X$ be a quasicompact and quasiseparated scheme. The assignments

$$
\mathcal{T} \in L_{\text {thick }}\left(\mathcal{D}_{\text {per }}(X)\right) \stackrel{\mu}{\longmapsto} Y(\mathcal{T})=\bigcup_{E \in \mathcal{T}} \operatorname{supph}_{X}(E)
$$

and

$$
Y \in L_{\text {open }}\left(X^{*}\right) \stackrel{\nu}{\longmapsto} \mathcal{T}(Y)=\left\{E \in \mathcal{D}_{\text {per }}(X) \mid \operatorname{supph}_{X}(E) \subseteq Y\right\}
$$

are mutually inverse lattice isomorphisms.

The next result says that there is a one-to-one correspondence between the tensor thick subcategories of perfect complexes and the tensor localizing subcategories of finite type of quasicoherent sheaves.

Theorem 27 (see Garkusha-Prest [5] for affine schemes). Let $X$ be a quasicompact and quasiseparated scheme. The assignments

$$
\mathcal{T} \in L_{\text {thick }}\left(\mathcal{D}_{\text {per }}(X)\right) \stackrel{\rho}{\longmapsto} \mathcal{S}=\left\{\mathcal{F} \in \mathrm{Q} \operatorname{coh}(X) \mid \operatorname{supp}_{X}(\mathcal{F}) \subseteq Y(\mathcal{T})\right\}
$$

and

$$
\mathcal{S} \in L_{\mathrm{f} . \text { loc }, \otimes}(X) \stackrel{\tau}{\longmapsto}\left\{E \in \mathcal{D}_{\text {per }}(X) \mid H_{n}(E) \in \mathcal{S} \text { for all } n \in \mathbb{Z}\right\}
$$

are mutually inverse lattice isomorphisms.

Proof. Consider the diagram

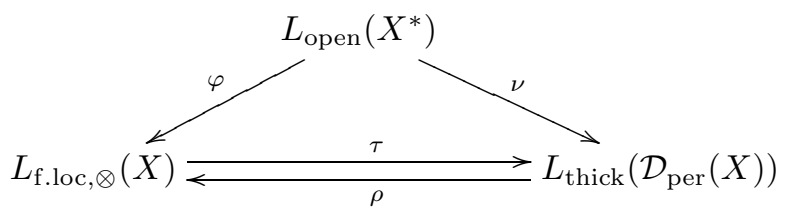

in which $\varphi, \nu$ are the lattice maps described in Theorems 25] and 26. Using the fact that $\operatorname{supph}_{X}(E)=\bigcup_{n \in \mathbb{Z}} \operatorname{supp}_{X}\left(H_{n}(E)\right), E \in \mathcal{D}_{\text {per }}(X)$, and Theorems 25, 26. we see that $\tau \varphi=\nu$ and $\rho \nu=\varphi$. Then $\rho \tau=\rho \nu \varphi^{-1}=\varphi \varphi^{-1}=1$ and $\tau \rho=\tau \varphi \nu^{-1}=\nu \nu^{-1}=1$.

\section{$\S 6$. The Zariski topology on $\mathrm{Sp}(\mathrm{X})$}

We are going to construct two maps

$$
\alpha: X \rightarrow \operatorname{Sp}(X) \text { and } \beta: \operatorname{Sp}(X) \rightarrow X .
$$

For any $P \in X$ there is an affine neighborhood $U=\operatorname{Spec} R$ of $P$. Let $E_{P}$ denote the injective hull of the quotient module $R / P$. Then $E_{P}$ is an indecomposable injective $R$-module. By Proposition 1, $\operatorname{Mod} R$ can be regarded as the quotient category $\mathrm{Q} \operatorname{coh}(X) / \mathcal{S}_{U}$ with $\mathcal{S}_{U}=\operatorname{Ker} j_{U}^{*}$, where $j_{U}: U \rightarrow X$ is the canonical injection. Therefore, $j_{U, *}: \operatorname{Mod} R \rightarrow \mathrm{Q} \operatorname{coh}(X)$ takes injectives to injectives. We set

$$
\alpha(P)=j_{U, *}\left(E_{P}\right) \in \operatorname{Sp}(X) .
$$

The definition of $\alpha$ does not depend on the choice of an affine neighborhood $U$. Indeed, let $P \in V=\operatorname{Spec} S$ with $S$ a commutative ring. Then $j_{U, *}\left(E_{P}\right) \cong j_{V, *}\left(E_{P}\right) \cong$ $j_{U \cap V, *}\left(E_{P}\right)$, so that these elements represent one and the same element in $\operatorname{Sp}(X)$. We denote it by $\mathcal{E}_{P}$. 
Now we define the map $\beta$. Let $X=\bigcup_{i=1}^{n} U_{i}$, where each $U_{i}=\operatorname{Spec} R_{i}$ is an affine scheme, and let $\mathcal{E} \in \operatorname{Sp}(X)$. Then $\mathcal{E}$ has no $\mathcal{S}_{U_{i}}$-torsion for some $i \leq n$, because $\bigcap_{i=1}^{n} \mathcal{S}_{U_{i}}=0$ and $\mathcal{E}$ is uniform. Since $\operatorname{Mod} R_{i}$ is equivalent to $\operatorname{Qcoh}(X) / \mathcal{S}_{U_{i}}, \mathcal{E}$ can be regarded as an indecomposable injective $R_{i}$-module. Set $P=P(\mathcal{E})$ to be the sum of annihilator ideals in $R_{i}$ of nonzero elements (equivalently, of nonzero submodules) of $\mathcal{E}$. Since $\mathcal{E}$ is uniform, the set of annihilator ideals of nonzero elements of $\mathcal{E}$ is closed under finite sums. It is easy to check (see $25,9.2]$ ) that $P(\mathcal{E})$ is a prime ideal. By construction, $P(\mathcal{E}) \in U_{i}$. Clearly, the definition of $P(\mathcal{E})$ does not depend on the choice of $U_{i}$ and $P\left(\mathcal{E}_{P}\right)=P$. Putting $\beta(\mathcal{E})=P(\mathcal{E})$, we see that $\beta \alpha=1_{X}$. In particular, $\alpha$ is an embedding of $X$ into $\operatorname{Sp}(X)$. We shall view this embedding as identification.

Given a commutative coherent ring $R$ and an indecomposable injective $R$-module $E \in \operatorname{Sp} R$, Prest [25, 9.6] observed that $E$ is elementarily equivalent to $E_{P(E)}$ in the first order language of modules. Translated from model-theoretic idioms to algebraic language, this fact says that every localizing subcategory of finite type $\mathcal{S} \in L_{\text {f.loc }}(\operatorname{Mod} R)$ is cogenerated by prime ideals. More precisely, there is a set $D \subset \operatorname{Spec} R$ such that $S \in \mathcal{S}$ if and only if $\operatorname{Hom}_{R}\left(S, E_{P}\right)=0$ for all $P \in D$. This fact was generalized to all commutative rings by Garkusha-Prest [5. Moreover, $D=\operatorname{Spec} R \backslash \bigcup_{S \in \mathcal{S}} \operatorname{supp}_{R}(S)$.

Proposition 28. Let $\mathcal{E} \in \mathrm{Sp}(X)$, and let $P(\mathcal{E}) \in X$ be the point defined above. Then $\mathcal{E}$ and $\mathcal{E}_{P(\mathcal{E})}$ are topologically indistinguishable in $\mathrm{Sp}_{\mathrm{fl}}(X)$. In other words, for every $\mathcal{S} \in L_{\text {f.loc }}(X)$, the sheaf $\mathcal{E}$ has no $\mathcal{S}$-torsion if and only if $\mathcal{E}_{P(\mathcal{E})}$ has no $\mathcal{S}$-torsion.

Proof. Let $U=\operatorname{Spec} R \subset X$ be such that $\mathcal{E}$ has no $\mathcal{S}_{U}$-torsion. Then $P(\mathcal{E}) \in U$ and $\mathcal{E}$ and $\mathcal{E}_{P(\mathcal{E})}$ have no $\mathcal{S}_{U}$-torsion. These can also be viewed as indecomposable injective $R$-modules, because $\mathrm{Q} \operatorname{coh}(X) / \mathcal{S}_{U} \cong \operatorname{Mod} R$ by Proposition 11. Denote $\mathcal{S}^{\prime}=\alpha_{X, U}(\mathcal{S}) \in$ $L_{\text {f.loc }}(\operatorname{Mod} R)$. Then $\mathcal{E}$ has no $\mathcal{S}$-torsion in $\mathrm{Qcoh}(X)$ if and only if $\mathcal{E}$ has no $\mathcal{S}^{\prime}$-torsion in $\operatorname{Mod} R$. Now our claim follows from [5, 3.5].

Corollary 29. If $\mathcal{S} \in L_{\mathrm{f} . \text { loc }, \otimes}(X)$, then $\mathcal{O}(\mathcal{S}) \cap X=Y(\mathcal{S})$, where

$$
Y(\mathcal{S})=\bigcup_{\mathcal{F} \in \mathcal{S}} \operatorname{supp}_{X}(\mathcal{F}) \in L_{\text {open }}\left(X^{*}\right) .
$$

Proof. If $\mathcal{E} \in \mathcal{O}(\mathcal{S})$ and $U=\operatorname{Spec} R \subset X$ is such that $\mathcal{E}$ has no $\mathcal{S}_{U}$-torsion, then $P(\mathcal{E}) \in U$ and $\mathcal{E}_{P(\mathcal{E})} \in \mathcal{O}(\mathcal{S})$ by Proposition 28, Let $\mathcal{S}^{\prime}=\alpha_{X, U}(\mathcal{S}) \in L_{\text {f.loc }}(\operatorname{Mod} R)$. We have $Y\left(\mathcal{S}^{\prime}\right):=\bigcup_{S \in \mathcal{S}} \operatorname{supp}_{R}(S) \subset Y(\mathcal{S})$. By the proof of Proposition 28, $\mathcal{E}_{P(\mathcal{E})}$ has $\mathcal{S}^{\prime}$-torsion. Then there is a finitely generated ideal $I \subset R$ such that $R / I \in \mathcal{S}^{\prime}$ and $\operatorname{Hom}_{R}\left(R / I, \mathcal{E}_{P(\mathcal{E})}\right) \neq 0$. From [5, 3.4] it follows that $P(\mathcal{E}) \in Y\left(\mathcal{S}^{\prime}\right)$, whence $\mathcal{O}(\mathcal{S}) \cap X \subset$ $Y(\mathcal{S})$.

Conversely, if $P \in Y(\mathcal{S}) \cap U$, then $\mathcal{E}_{P}$ has $\mathcal{S}^{\prime}$-torsion by [5. 3.4]. Therefore, $\mathcal{E}_{P} \in$ $\mathcal{O}\left(\mathcal{S}^{\prime}\right) \subset \mathcal{O}(\mathcal{S})$. It follows that $\mathcal{O}(\mathcal{S}) \cap X \supset Y(\mathcal{S})$.

Proposition 30 (cf. [5, 3.7]). Let $X$ be a quasicompact and quasiseparated scheme. Then the maps

$$
Y \in L_{\text {open }}\left(X^{*}\right) \stackrel{\sigma}{\mapsto} \mathcal{O}_{Y}=\{\mathcal{E} \in \mathrm{Sp}(X) \mid P(\mathcal{E}) \in Y\}
$$

and

$$
\mathcal{O} \in L_{\mathrm{open}}\left(\mathrm{Sp}_{\mathrm{ff}, \otimes}(X)\right) \stackrel{\mathcal{\varepsilon}}{\mapsto} Y_{\mathcal{O}}=\left\{P(\mathcal{E}) \in X^{*} \mid \mathcal{E} \in \mathcal{O}\right\}=\mathcal{O} \cap X^{*}
$$

induce a one-to-one correspondence between the lattices of open sets of $X^{*}$ and those of $\mathrm{Sp}_{\mathrm{ff}, \otimes}(X)$.

Proof. Let $\mathcal{S}(Y)=\left\{\mathcal{F} \in \operatorname{Qcoh}(X) \mid \operatorname{supp}_{X}(\mathcal{F}) \subseteq Y\right\} \in L_{\text {f.loc }, \otimes}(X)$; then $Y=Y(\mathcal{S}(Y))$ by the classification theorem, and $\mathcal{O}(\mathcal{S}(Y)) \cap X=Y$ by Corollary 29] It follows that $\mathcal{O}(\mathcal{S}(Y)) \subseteq \mathcal{O}_{Y}$. On the other hand, if $\mathcal{E} \in \mathcal{O}_{Y}$, then the proof of Corollary 29] shows that 
$\mathcal{E}_{P(\mathcal{E})} \in \mathcal{O}(\mathcal{S}(Y))$. Proposition 28 implies that $\mathcal{E} \in \mathcal{O}(\mathcal{S}(Y))$, whence $\mathcal{O}(\mathcal{S}(Y)) \supseteq \mathcal{O}_{Y}$. We see that $\mathcal{O}_{Y}=\mathcal{O}(\mathcal{S}(Y)) \in L_{\mathrm{open}}\left(\mathrm{Sp}_{\mathrm{fl}, \otimes}(X)\right)$.

Let $\mathcal{O} \in L_{\mathrm{open}}\left(\mathrm{Sp}_{\mathrm{fl}, \otimes}(X)\right)$. By Theorem 19, there is a unique $\mathcal{S} \in L_{\mathrm{f} . \text { loc }, \otimes}(X)$ such that $\mathcal{O}=\mathcal{O}(\mathcal{S})$. By Corollary 29, $\mathcal{O} \cap X=Y(\mathcal{S})=Y_{\mathcal{O}}$, so that $Y_{\mathcal{O}} \in L_{\text {open }}\left(X^{*}\right)$. Now it is easy to verify that $Y_{\mathcal{O}_{Y}}=Y$ and $\mathcal{O}_{Y_{\mathcal{O}}}=\mathcal{O}$.

We note that a subset $Y \subset X^{*}$ is open and quasicompact in $X^{*}$ if and only if $X \backslash Y$ is an open and quasicompact subset in $X$.

Proposition 31. An open subset $\mathcal{O} \in L_{\mathrm{open}}\left(\mathrm{Sp}_{\mathrm{fl}, \otimes}(X)\right)$ is quasicompact if and only if it is of the form $\mathcal{O}=\mathcal{O}(\mathcal{S}(Y))$, where $Y$ is an open and quasicompact subset in $X^{*}$. The space $\mathrm{Sp}_{\mathrm{fl}, \otimes}(X)$ is quasicompact, the quasicompact open subsets are closed under finite intersections and form an open basis, and every nonempty irreducible closed subset has a generic point.

Proof. Let $\mathcal{O} \in L_{\mathrm{open}}\left(\mathrm{Sp}_{\mathrm{fl}, \otimes}(X)\right)$ be quasicompact. By Theorem 19, there is a unique $\mathcal{S} \in L_{\text {f.loc }, \otimes}(X)$ such that $\mathcal{O}=\mathcal{O}(\mathcal{S})$. By the classification theorem, $\mathcal{S}=\mathcal{S}(Y)=$ $\sqrt{ }\left(\bigcup_{I} \mathcal{S}\left(Y_{i}\right)\right)$, where $Y=\bigcup_{\mathcal{F} \in \mathcal{S}} \operatorname{supp}_{X}(\mathcal{F}) \in L_{\text {open }}\left(X^{*}\right)$, each $Y_{i}$ is such that $X \backslash Y_{i}$ is an open and quasicompact subset of $X$, and $Y=\bigcup_{I} Y_{i}$. Then $\mathcal{O}=\bigcup_{I} \mathcal{O}\left(\mathcal{S}\left(Y_{i}\right)\right)$. Since $\mathcal{O}$ is quasicompact, there is a finite subset $J \subset I$ such that $\mathcal{O}=\bigcup_{J} \mathcal{O}\left(\mathcal{S}\left(Y_{i}\right)\right)=$ $\mathcal{O}\left(\sqrt{ }\left(\bigcup_{J} \mathcal{S}\left(Y_{i}\right)\right)\right)=\mathcal{O}\left(\mathcal{S}\left(\bigcup_{J} Y_{i}\right)\right)$. Since $X$ is spectral, $X \backslash\left(\bigcup_{J} Y_{i}\right)=\bigcap_{J}\left(X \backslash Y_{i}\right)$ is an open and quasicompact subset in $X$.

Conversely, let $\mathcal{O}=\mathcal{O}(\mathcal{S}(Y)$ ), where $X \backslash Y$ is an open and quasicompact subset in $X$, and let $\mathcal{O}=\bigcup_{I} \mathcal{O}_{i}$ with $\mathcal{O}_{i} \in L_{\mathrm{open}}\left(\operatorname{Sp}_{\mathrm{fl}, \otimes}(X)\right)$. By Theorem 19, there are unique $\mathcal{S}_{i} \in$ $L_{\text {f.loc }, \otimes}(X)$ such that $\mathcal{O}_{i}=\mathcal{O}\left(\mathcal{S}_{i}\right)$ and $\mathcal{S}(Y)=\sqrt{ }\left(\bigcup_{I} \mathcal{S}_{i}\right)$. We set $Y_{i}=\bigcup_{\mathcal{F} \in \mathcal{S}_{i}} \operatorname{supp}_{X}(\mathcal{F})$ for each $i \in I$. By Lemma 23 and the classification theorem, we have $Y=\bigcup_{I} Y_{i}$. Since $Y$ is quasicompact in $X^{*}$, there is a finite subset $J \subset I$ such that $Y=\bigcup_{J} Y_{i}$. It follows that $\mathcal{S}(Y)=\sqrt{ }\left(\bigcup_{J} \mathcal{S}_{i}\right)$ and $\mathcal{O}=\bigcup_{J} \mathcal{O}_{i}$.

The space $\mathrm{Sp}_{\mathrm{fl}, \otimes}(X)$ is quasicompact because it equals $\mathcal{O}\left(\mathcal{S}\left(X^{*}\right)\right)$ and $X^{*}$ is quasicompact. The quasicompact open subsets are closed under finite intersections, because $\mathcal{O}\left(\mathcal{S}\left(Y_{1}\right)\right) \cap \mathcal{O}\left(\mathcal{S}\left(Y_{2}\right)\right)=\mathcal{O}\left(\mathcal{S}\left(Y_{1} \cap Y_{2}\right)\right)$ with $Y_{1}, Y_{2}$ open and quasicompact in $X^{*}$. Since $\mathcal{O}(\mathcal{S}(Y))=\bigcup_{I} \mathcal{O}\left(\mathcal{S}\left(Y_{i}\right)\right)$, where $Y=\bigcup_{I} Y_{i}$ and each $Y_{i}$ is an open and quasicompact subset in $X^{*}$, the quasicompact open subsets also form an open basis.

Finally, from Corollary 29 it follows that a subset $U$ of $\mathrm{Sp}_{\mathrm{fl}, \otimes}(X)$ is closed and irreducible if and only if so is $\widehat{U}:=U \cap X^{*}$. Since $X^{*}$ is spectral, $\widehat{U}$ has a generic point $P$. The point $\mathcal{E}_{P} \in U$ is generic.

In general, the space $\mathrm{Sp}_{\mathrm{fl}, \otimes}(X)$ is not $T_{0}$ (see [3]); nevertheless, we give the same definition for $\left(\mathrm{Sp}_{\mathrm{fl}, \otimes}(X)\right)^{*}$ as for spectral spaces and denote this space by $\operatorname{Sp}_{\text {zar }}(X)$. By definition, $\mathcal{Q} \in L_{\text {open }}\left(\operatorname{Sp}_{\text {zar }}(X)\right)$ if and only if $\mathcal{Q}=\bigcup_{I} \mathcal{Q}_{i}$ with each $\mathcal{Q}_{i}$ having a quasicompact and open complement in $\mathrm{Sp}_{\mathrm{ff}, \otimes}(X)$. The topology on $\mathrm{Sp}_{\mathrm{zar}}(X)$ will also be referred to as the Zariski topology. Notice that the Zariski topology on $\mathrm{Sp}_{\mathrm{zar}}(\mathrm{Spec} R)$, where $R$ is coherent, coincides with the Zariski topology on the injective spectrum $\operatorname{Sp} R$ in the sense of Prest [25].

Theorem 32 (cf. Garkusha-Prest [3, 4, 5]). Let X be a quasicompact and quasiseparated scheme. The space $X$ is dense and is a retract in $\mathrm{Sp}_{\mathrm{zar}}(X)$. A left inverse to the embedding $X \hookrightarrow \mathrm{Sp}_{\mathrm{zar}}(X)$ takes $\mathcal{E} \in \mathrm{Sp}_{\mathrm{zar}}(X)$ to $P(\mathcal{E}) \in X$. Moreover, $\mathrm{Sp}_{\mathrm{zar}}(X)$ is quasicompact, the basic open subsets $\mathcal{Q}$, where $\operatorname{Sp}(X) \backslash \mathcal{Q}$ is a quasicompact and open subset in $\mathrm{Sp}_{\mathrm{fl}, \otimes}(X)$, are quasicompact, the intersection of two quasicompact open subsets is quasicompact, and every nonempty irreducible closed subset has a generic point.

Proof. Let $\mathcal{Q} \in L_{\mathrm{open}}\left(\mathrm{Sp}_{\mathrm{zar}}(X)\right)$ be such that $\mathcal{O}:=\mathrm{Sp}(X) \backslash \mathcal{Q}$ is a quasicompact and open subset in $\operatorname{Sp}_{\mathrm{fl}, \otimes}(X)$, and let $Y=\mathcal{O} \cap X$ and $D=X \backslash Y=\mathcal{Q} \cap X$. Since $Y$ is a 
quasicompact subset in $X^{*}$, we see that $D$ is a quasicompact subset in $X$. Observe that $\mathcal{O}=\mathcal{O}\left(\mathcal{S}_{D}\right)$, where $\mathcal{S}_{D}=\left\{\mathcal{F} \in \mathrm{Q} \operatorname{coh}(X)|\mathcal{F}|_{D}=0\right\}$. Clearly, $X$ is dense in $\operatorname{Sp}_{\text {zar }}(X)$, and $\alpha: X \rightarrow \operatorname{Sp}_{\text {zar }}(X)$ is a continuous map.

The map $\beta: \operatorname{Sp}_{\text {zar }}(X) \rightarrow X, \mathcal{E} \mapsto P(\mathcal{E})$, is left inverse to $\alpha$. Obviously, $\beta$ is continuous. Thus, $X$ is a retract of $\operatorname{Sp}_{\text {zar }}(X)$.

We show that the basic open set $\mathcal{Q}$ is quasicompact. Let $\mathcal{Q}=\bigcup_{i \in \Omega} \mathcal{Q}_{i}$, whence each $\operatorname{Sp}(X) \backslash \mathcal{Q}_{i}$ is a quasicompact and open subset in $\mathrm{Sp}_{\mathrm{fl}, \otimes}(X)$, and let $D_{i}:=\mathcal{Q}_{i} \cap X$. Since $D$ is quasicompact, we have $D=\bigcup_{i \in \Omega_{0}} D_{i}$ for some finite subset $\Omega_{0} \subset \Omega$.

Assume $\mathcal{E} \in \mathcal{Q} \backslash \bigcup_{i \in \Omega_{0}} \mathcal{Q}_{i}$. By Proposition 30, $P(\mathcal{E}) \in \mathcal{Q} \cap X=D=\bigcup_{i \in \Omega_{0}} D_{i}$. Proposition 30 implies that $\mathcal{E} \in \mathcal{Q}_{i_{0}}$ for some $i_{0} \in \Omega_{0}$, a contradiction. So, $\mathcal{Q}$ is quasicompact. Also, it follows that the intersection of two quasicompact open subsets is quasicompact and that $\mathrm{Sp}_{\mathrm{zar}}(X)$ is quasicompact.

Finally, from Corollary 29 it follows that a subset $U$ of $\operatorname{Sp}_{\text {zar }}(X)$ is closed and irreducible if and only if so is $\widehat{U}:=U \cap X$. Since $X$ is spectral, $\widehat{U}$ has a generic point $P$. The point $\mathcal{E}_{P} \in U$ is generic.

Corollary 33. Let $X$ be a quasicompact and quasiseparated scheme. Then

$$
\mathrm{Sp}_{\mathrm{zar}}(X)=\left(\mathrm{Sp}_{\mathrm{fl}, \otimes}(X)\right)^{*} \text { and } \mathrm{Sp}_{\mathrm{fl}, \otimes}(X)=\left(\mathrm{Sp}_{\mathrm{zar}}(X)\right)^{*} \text {. }
$$

Though the space $\operatorname{Sp}_{\mathrm{zar}}(X)$ is strictly larger than $X$ in general (see [3]), their lattices of open subsets are isomorphic. More precisely, Proposition 30 shows that the maps

$$
D \in L_{\text {open }}(X) \mapsto \mathcal{Q}_{D}=\{\mathcal{E} \in \operatorname{Sp}(X) \mid P(\mathcal{E}) \in D\}
$$

and

$$
\mathcal{Q} \in L_{\text {open }}\left(\operatorname{Sp}_{\text {zar }}(X)\right) \mapsto D_{\mathcal{Q}}=\{P(\mathcal{E}) \in X \mid \mathcal{E} \in \mathcal{Q}\}=\mathcal{Q} \cap X
$$

induce a one-to-one correspondence between the lattices of open sets of $X$ and those of $\operatorname{Sp}_{\mathrm{zar}}(X)$. Moreover, sheaves do not see any difference between $X$ and $\operatorname{Sp}_{\mathrm{zar}}(X)$. Namely, the following is true.

Proposition 34. Let $X$ be a quasicompact and quasiseparated scheme. Then the maps of topological spaces $\alpha: X \rightarrow \mathrm{Sp}_{\mathrm{zar}}(X)$ and $\beta: \mathrm{Sp}_{\mathrm{zar}}(X) \rightarrow X$ induce isomorphisms

$$
\beta_{*}: \operatorname{Sh}\left(\operatorname{Sp}_{\mathrm{zar}}(X)\right) \stackrel{\cong}{\longrightarrow} \operatorname{Sh}(X), \quad \alpha_{*}: \operatorname{Sh}(X) \stackrel{\cong}{\longrightarrow} \operatorname{Sh}\left(\mathrm{Sp}_{\mathrm{zar}}(X)\right)
$$

of categories of sheaves.

Proof. Since $\beta \alpha=1$, it follows that $\beta_{*} \alpha_{*}=1$. By definition, $\beta_{*}(\mathcal{F})(D)=\mathcal{F}\left(\mathcal{Q}_{D}\right)$ for any $\mathcal{F} \in S h\left(\operatorname{Sp}_{\text {zar }}(X)\right)$; next, $D \in L_{\text {open }}(X)$ and $\alpha_{*}(\mathcal{G})(\mathcal{Q})=\mathcal{G}\left(D_{\mathcal{Q}}\right)$ for any $\mathcal{G} \in S h(X)$ and $\mathcal{Q} \in L_{\text {open }}\left(\operatorname{Sp}_{\text {zar }}(X)\right)$. We have

$$
\alpha_{*} \beta_{*}(\mathcal{F})(\mathcal{Q})=\beta_{*}(\mathcal{F})\left(D_{\mathcal{Q}}\right)=\mathcal{F}\left(\mathcal{Q}_{D_{\mathcal{Q}}}\right)=\mathcal{F}(\mathcal{Q}) .
$$

Thus, $\alpha_{*} \beta_{*}=1$, so that $\alpha_{*}, \beta_{*}$ are mutually inverse isomorphisms.

Let $\mathcal{O}_{\mathrm{Sp}_{\mathrm{zar}}(X)}$ be the sheaf of commutative rings $\alpha_{*}\left(\mathcal{O}_{X}\right)$; clearly, $\left(\operatorname{Sp}_{\mathrm{zar}}(X), \mathcal{O}_{\mathrm{Sp}_{\mathrm{zar}}(X)}\right)$ is a locally ringed space. If we set $\alpha^{\sharp}: \mathcal{O}_{\mathrm{Sp}_{\mathrm{zar}}(X)} \rightarrow \alpha_{*} \mathcal{O}_{X}$ and $\beta^{\sharp}: \mathcal{O}_{X} \rightarrow \beta_{*} \mathcal{O}_{\mathrm{Sp}_{\mathrm{zar}}(X)}$ to be the identity maps, then the map of locally ringed spaces

$$
\left(\alpha, \alpha^{\sharp}\right):\left(X, \mathcal{O}_{X}\right) \rightarrow\left(\operatorname{Sp}_{\mathrm{zar}}(X), \mathcal{O}_{\mathrm{Sp}_{\mathrm{zar}}(X)}\right)
$$

is right inverse to

$$
\left(\beta, \beta^{\sharp}\right):\left(\operatorname{Sp}_{\text {zar }}(X), \mathcal{O}_{\mathrm{Sp}_{\text {zar }}(X)}\right) \rightarrow\left(X, \mathcal{O}_{X}\right) .
$$

Observe that it is not a scheme in general, because $\mathrm{Sp}_{\text {zar }}(X)$ is not a $T_{0}$-space. Proposi-

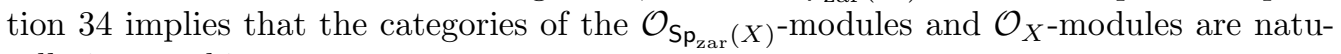
rally isomorphic. 


\section{$\S 7$. The PRIME SPECTRUM OF AN IDEAL LATTICE}

Inspired by a recent paper of Balmer (see 24]), Buan, Krause, and Solberg 2 introduced the notion of an ideal lattice and studied its prime ideal spectrum. Applications arise in Abelian or triangulated tensor categories.

Definition (Buan, Krause, and Solberg [2]). An ideal lattice is a partially ordered set $L=(L, \leq)$ together with an associative multiplication $L \times L \rightarrow L$ with the following properties.

(L1) The poset $L$ is a complete lattice; that is,

$$
\sup A=\bigvee_{a \in A} a \text { and } \inf A=\bigwedge_{a \in A} a
$$

exist in $L$ for every subset $A \subseteq L$.

(L2) The lattice $L$ is compactly generated; that is, every element in $L$ is the supremum of a set of compact elements. (An element $a \in L$ is compact if for all $A \subseteq L$ with $a \leq \sup A$ there exists some finite $A^{\prime} \subseteq A$ with $a \leq \sup A^{\prime}$.)

(L3) For all $a, b, c \in L$ we have

$$
a(b \vee c)=a b \vee a c \quad \text { and } \quad(a \vee b) c=a c \vee b c .
$$

(L4) The element $1=\sup L$ is compact, and $1 a=a=a 1$ for all $a \in L$.

(L5) The product of two compact elements is again compact.

A morphism $\varphi: L \rightarrow L^{\prime}$ of ideal lattices is a map satisfying

$$
\begin{gathered}
\varphi\left(\bigvee_{a \in A} a\right)=\bigvee_{a \in A} \varphi(a) \text { for } A \subseteq L, \\
\varphi(1)=1 \text { and } \varphi(a b)=\varphi(a) \varphi(b) \text { for } a, b \in L .
\end{gathered}
$$

Let $L$ be an ideal lattice. Following 2, we define the spectrum of prime elements in $L$. An element $p \neq 1$ in $L$ is prime if $a b \leq p$ implies $a \leq p$ or $b \leq p$ for all $a, b \in L$. We denote by Spec $L$ the set of prime elements in $L$ and, for each $a \in L$, define

$$
V(a)=\{p \in \operatorname{Spec} L \mid a \leq p\} \quad \text { and } \quad D(a)=\{p \in \operatorname{Spec} L \mid a \not \leq p\} .
$$

The subsets of Spec $L$ of the form $V(a)$ are closed under forming arbitrary intersections and finite unions. More precisely,

$$
V\left(\bigvee_{i \in \Omega} a_{i}\right)=\bigcap_{i \in \Omega} V\left(a_{i}\right) \text { and } \quad V(a b)=V(a) \cup V(b) .
$$

Thus, we obtain the Zariski topology on $\operatorname{Spec} L$ by declaring a subset of $\operatorname{Spec} L$ to be closed if it is of the form $V(a)$ for some $a \in L$. The set Spec $L$ endowed with this topology is called the prime spectrum of $L$. Note that the sets of the form $D(a)$ with compact $a \in L$ form a basis of open sets. The prime spectrum Spec $L$ of an ideal lattice $L$ is spectral [2, 2.5].

There is a close relationship between spectral spaces and ideal lattices. Given a topological space $X$, we denote by $L_{\text {open }}(X)$ the lattice of open subsets of $X$ and consider the multiplication map

$$
L_{\text {open }}(X) \times L_{\text {open }}(X) \rightarrow L_{\text {open }}(X), \quad(U, V) \mapsto U V=U \cap V .
$$

The lattice $L_{\text {open }}(X)$ is complete.

The following result, which appeared in [2], is part of the Stone duality theorem (see, e.g., [26]). 
Proposition 35. Let $X$ be a spectral space. Then $L_{\mathrm{open}}(X)$ is an ideal lattice. Moreover, the map

is a homeomorphism.

$$
X \rightarrow \operatorname{Spec} L_{\text {open }}(X), \quad x \mapsto X \backslash \overline{\{x\}},
$$

The next statement is deduced from the classification theorem.

Proposition 36. Let $X$ be a quasicompact and quasiseparated scheme. Then $L_{\mathrm{f} . \mathrm{loc}, \otimes}(X)$ is an ideal lattice.

Proof. The space $X$ is spectral. Thus, $X^{*}$ is spectral, and $L_{\mathrm{open}}\left(X^{*}\right)$ is an ideal lattice by Proposition 35. By the classification theorem, we have the isomorphism $L_{\text {open }}\left(X^{*}\right) \cong$ $L_{\mathrm{f} . l o c, \otimes}(X)$. Therefore, $L_{\mathrm{f} . \text { loc }, \otimes}(X)$ is an ideal lattice.

Proposition 31 implies that $\mathcal{S} \in L_{\mathrm{f} . \text { loc }, \otimes}(X)$ is compact if and only if $\mathcal{S}=\mathcal{S}(Y)$ with $Y \in L_{\text {open }}\left(X^{*}\right)$ compact.

Corollary 37. Let $X$ be a quasicompact and quasiseparated scheme. The points of $\operatorname{Spec} L_{\mathrm{f} . l o c,} \otimes(X)$ are the $\cap$-irreducible tensor localizing subcategories of finite type in $\mathrm{Q} \operatorname{coh}(X)$, and the map

$$
f: X^{*} \rightarrow \operatorname{Spec} L_{\text {f.loc }, \otimes}(X), \quad P \mapsto \mathcal{S}_{P}=\left\{\mathcal{F} \in \operatorname{Q} \operatorname{coh}(X) \mid \mathcal{F}_{P}=0\right\}
$$

is a homeomorphism of spaces.

Proof. This is a consequence of the classification theorem and Propositions 35 and 36 .

\section{§8. RECONSTRUCTING QUASICOMPACT, QUASISEPARATED SCHEMES}

Let $X$ be a quasicompact and quasiseparated scheme. We write $\operatorname{Spec}(\mathrm{Q} \operatorname{coh}(X)):=$ $\left(\operatorname{Spec} L_{\text {f.loc }, \otimes}(X)\right)^{*}$ and $\operatorname{supp}(\mathcal{F}):=\{\mathcal{P} \in \operatorname{Spec}(\mathrm{Q} \operatorname{coh}(X)) \mid \mathcal{F} \notin \mathcal{P}\}$ for $\mathcal{F} \in \operatorname{Qcoh}(X)$. From Corollary 37 it follows that

$$
\operatorname{supp}_{X}(\mathcal{F})=f^{-1}(\operatorname{supp}(\mathcal{F})) \text {. }
$$

Following 24, 2], we define a structure sheaf on $\operatorname{Spec}(\mathrm{Q} \operatorname{coh}(X))$ as follows. For an open subset $U \subseteq \operatorname{Spec}(\mathrm{Q} \operatorname{coh}(X))$, we put

$$
\mathcal{S}_{U}=\{\mathcal{F} \in \operatorname{Qcoh}(X) \mid \operatorname{supp}(\mathcal{F}) \cap U=\varnothing\}
$$

and observe that $\mathcal{S}_{U}=\left\{\mathcal{F} \mid \mathcal{F}_{P}=0\right.$ for all $\left.P \in f^{-1}(U)\right\}$ is a tensor localizing subcategory. We obtain a presheaf of rings on $\operatorname{Spec}(\mathrm{Q} \operatorname{coh}(X))$ :

$$
U \mapsto \operatorname{End}_{\mathrm{Qcoh}(X) / \mathcal{S}_{U}}\left(\mathcal{O}_{X}\right) .
$$

If $V \subseteq U$ are open subsets, then the restriction map

$$
\operatorname{End}_{\mathrm{Q} c o h}(X) / \mathcal{S}_{U}\left(\mathcal{O}_{X}\right) \rightarrow \operatorname{End}_{\mathrm{Qcoh}(X) / \mathcal{S}_{V}}\left(\mathcal{O}_{X}\right)
$$

is induced by the quotient functor $\mathrm{Q} \operatorname{coh}(X) / \mathcal{S}_{U} \rightarrow \mathrm{Q} \operatorname{coh}(X) / \mathcal{S}_{V}$. The sheafification is called the structure sheaf of $\mathrm{Q} \operatorname{coh}(X)$ and is denoted by $\mathcal{O}_{\mathrm{Qcoh}(X)}$. Next, let $\mathcal{P} \in$ $\operatorname{Spec}(\operatorname{Qcoh}(X))$, and let $P:=f^{-1}(\mathcal{P})$. There is an affine neighborhood Spec $R$ of $P$. We have

$$
\mathcal{O}_{\mathrm{Qcoh}(X), \mathcal{P}} \cong \lim _{\overrightarrow{\mathcal{P} \in V}} \operatorname{End}_{\mathrm{Mod} R / \mathcal{S}_{V}}(R) \cong R_{P} \cong \mathcal{O}_{X, P}
$$

The second isomorphism follows from [5, §8]. We see that each stalk $\mathcal{O}_{\mathrm{Q} \operatorname{coh}(X), \mathcal{P}}$ is a commutative ring. We claim that $\mathcal{O}_{\mathrm{Q}} \operatorname{coh}(X)$ is a sheaf of commutative rings. Indeed, let $a, b \in \mathcal{O}_{\mathrm{Qcoh}(X)}(U)$, where $U \in L_{\text {open }}(\operatorname{Spec}(\mathrm{Q} \operatorname{coh}(X)))$. For all $\mathcal{P} \in U$ we have $\varrho_{\mathcal{P}}^{U}(a b)=\varrho_{\mathcal{P}}^{U}(b a)$, where $\varrho_{\mathcal{P}}^{U}: \mathcal{O}_{\mathrm{Q} \operatorname{coh}(X)}(U) \rightarrow \mathcal{O}_{\mathrm{Qcoh}(X), \mathcal{P}}$ is the natural homomorphism. Since $\mathcal{O}_{\mathrm{Qcoh}(X)}$ is a sheaf, it follows that $a b=b a$. 
The next theorem says that the Abelian category $\mathrm{Q} \operatorname{coh}(X)$ contains all the necessary information to reconstruct the scheme $\left(X, \mathcal{O}_{X}\right)$.

Theorem 38 (Reconstruction; cf. Rosenberg [11). Let $X$ be a quasicompact and quasiseparated scheme. The map of Corollary 37 induces an isomorphism of ringed spaces

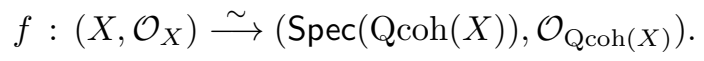

Proof. The proof is similar to that of [2, 8.3; 9.4]. Fix an open subset $U \subseteq \operatorname{Spec}(\mathrm{Q} \operatorname{coh}(X))$ and consider the functor

$$
F: \operatorname{Qcoh}(X) \stackrel{\left.(-)\right|_{f^{-1}(U)}}{\longrightarrow} \mathrm{Qcoh} f^{-1}(U) .
$$

We claim that $F$ annihilates $\mathcal{S}_{U}$. In fact, $\mathcal{F} \in \mathcal{S}_{U}$ implies $f^{-1}(\operatorname{supp}(\mathcal{F})) \cap f^{-1}(U)=\varnothing$, and therefore $\operatorname{supp}_{X}(\mathcal{F}) \cap f^{-1}(U)=\varnothing$. Thus, $\mathcal{F}_{P}=0$ for all $P \in f^{-1}(U)$, whence $F(\mathcal{F})=0$. It follows that $F$ factors through $\mathrm{Q} \operatorname{coh}(X) / \mathcal{S}_{U}$ and induces a map $\operatorname{End}_{\mathrm{Qcoh}(X) / \mathcal{S}_{U}}\left(\mathcal{O}_{X}\right) \rightarrow$ $\mathcal{O}_{X}\left(f^{-1}(U)\right)$, which extends to a map $\mathcal{O}_{\mathrm{Qcoh}(X)}(U) \rightarrow \mathcal{O}_{X}\left(f^{-1}(U)\right)$. This yields a morphism of sheaves $f^{\sharp}: \mathcal{O}_{\mathrm{Q}} \operatorname{coh}(X) \rightarrow f_{*} \mathcal{O}_{X}$.

By the above, $f^{\sharp}$ induces an isomorphism $f_{P}^{\sharp}: \mathcal{O}_{\mathrm{Q} \operatorname{coh}(X), f(P)} \rightarrow \mathcal{O}_{X, P}$ at each point $P \in X$. We conclude that $f_{P}^{\sharp}$ is an isomorphism. It follows that $f$ is an isomorphism of ringed spaces if the map $f: X \rightarrow \operatorname{Spec}(\mathrm{Q} \operatorname{coh}(X))$ is a homeomorphism. This last condition is a consequence of Propositions 35 36 and Corollary 37.

\section{§9. Coherent schemes}

We end the paper with introducing coherent schemes. These lie between Noetherian and quasicompact, quasiseparated schemes and generalize commutative coherent rings. We want to obtain classification and reconstruction results for such schemes.

Definition. A scheme $X$ is locally coherent if it can be covered by open affine subsets $\operatorname{Spec} R_{i}$, where each $R_{i}$ is a coherent ring. $X$ is coherent if it is locally coherent, quasicompact, and quasiseparated.

A trivial example of a coherent scheme is $\operatorname{Spec} R$ with $R$ a coherent ring. There are many coherent rings. For instance, let $R$ be a Noetherian ring, and let $X$ be any (possibly infinite) set of commuting indeterminates. Then the polynomial ring $R[X]$ is coherent. As a note of caution, however, we should mention that, in general, the coherence of a ring $R$ does not imply that of $R[x]$ for one variable $x$. In fact, if $R$ is a countable product of the polynomial ring $\mathbb{Q}[y, z]$, the ring $R$ is coherent, but $R[x]$ is not coherent by a result of Soublin [27. For any finitely generated ideal $I$ of a coherent ring $R$, the quotient ring $R / I$ is coherent.

If $R$ is a coherent ring such that the polynomial ring $R\left[x_{1}, \ldots, x_{n}\right]$ is coherent, then the projective $n$-space $\mathbb{P}_{R}^{n}=\operatorname{Proj} R\left[x_{0}, \ldots, x_{n}\right]$ over $R$ is a coherent scheme. Indeed, $\mathbb{P}_{R}^{n}$ is quasicompact and quasiseparated by [4, 5.1] and is covered by Spec $R\left[x_{0} / x_{i}, \ldots, x_{n} / x_{i}\right]$ with each $R\left[x_{0} / x_{i}, \ldots, x_{n} / x_{i}\right]$ coherent by assumption.

Below we shall need the following result.

Theorem 39 (Herzog [12, Krause [13). Let $\mathcal{C}$ be a locally coherent Grothendieck category. There is a bijective correspondence between the Serre subcategories $\mathcal{P}$ of coh $\mathcal{C}$ and the localizing subcategories $\mathcal{S}$ of $\mathcal{C}$ of finite type. This correspondence is given by the functions

$$
\begin{aligned}
\mathcal{P} \longmapsto \overrightarrow{\mathcal{P}} & =\left\{\underline{\left.\lim C_{i} \mid C_{i} \in \mathcal{P}\right\},}\right. \\
\mathcal{S} & \longmapsto \mathcal{S} \cap \operatorname{coh} \mathcal{C},
\end{aligned}
$$

which are mutually inverse. 
Proposition 40. Let $X$ be a quasicompact and quasiseparated scheme. Then $X$ is a coherent scheme if and only if $\operatorname{coh}(X)$ is an Abelian category, or equivalently, $\mathrm{Qcoh}(X)$ is a locally coherent Grothendieck category.

Proof. Suppose $X$ is a coherent scheme. We must show that every finitely generated subobject $\mathcal{F}$ of a finitely presented object $\mathcal{G}$ is finitely presented. From [15, I.6.9.10] and Proposition 7 it follows that $\mathcal{F} \in \operatorname{fg}(\mathrm{Q} \operatorname{coh}(X))$ if and only if it is locally finitely generated.

For any $P \in X$, there is an open subset $U$ of $P$ and an exact sequence

$$
\left.\mathcal{O}_{U}^{n} \rightarrow \mathcal{O}_{U}^{m} \rightarrow \mathcal{G}\right|_{U} \rightarrow 0 .
$$

By assumption, there is an affine neighborhood $\operatorname{Spec} R$ of $P$ with $R$ a coherent ring. Let $f \in R$ be such that $P \in D(f) \subseteq \operatorname{Spec} R \cap U$, where $D(f)=\{Q \in \operatorname{Spec} R \mid f \notin Q\}$. Since $\mathcal{O}_{X}(D(f))=\mathcal{O}_{R}(D(f))=R_{f}$, we get an exact sequence

$$
\left.\mathcal{O}_{R_{f}}^{n} \rightarrow \mathcal{O}_{R_{f}}^{m} \rightarrow \mathcal{G}\right|_{D(f)} \rightarrow 0 .
$$

If $R$ is a coherent ring, then so is $R_{f}$.

There is an open neighborhood $V$ of $P$ and an epimorphism $\left.\mathcal{O}_{V}^{k} \rightarrow \mathcal{F}\right|_{V}, k \in \mathbb{N}$. Without loss of generality, we may assume that $V=D(f)$ for some $f \in R$. It follows that $\left.\left.\mathcal{F}\right|_{D(f)} \subset \mathcal{G}\right|_{D(f)}$ is a finitely presented $\mathcal{O}_{R_{f}}$-module, because $R_{f}$ is a coherent ring. Therefore, $\mathcal{F}$ is locally finitely presented, whence $\mathcal{F} \in \mathrm{fp}(\mathrm{Qcoh}(X))$.

Now suppose that $\mathrm{Q} \operatorname{coh}(X)$ is a locally coherent Grothendieck category. Given $P \in X$ and an affine neighborhood $\operatorname{Spec} R$ of $P$, we want to show that $R$ is a coherent ring. The localizing subcategory $\mathcal{S}=\left\{\mathcal{F}|\mathcal{F}|_{\text {Spec } R}=0\right\}$ is of finite type; therefore, $\mathrm{Q} \operatorname{coh}(X) / \mathcal{S}$ is a locally coherent Grothendieck category. From Proposition 1 it follows that $\operatorname{Mod} R \cong$ $\mathrm{Q} \operatorname{coh}(\operatorname{Spec} R) \cong \mathrm{Q} \operatorname{coh}(X) / \mathcal{S}$ is a locally coherent Grothendieck category, whence $R$ is coherent.

Theorem 41 (Classification). Let $X$ be a coherent scheme. Then the maps

$$
V \mapsto \mathcal{S}=\left\{\mathcal{F} \in \operatorname{coh}(X) \mid \operatorname{supp}_{X}(\mathcal{F}) \subseteq V\right\}
$$

and

$$
\mathcal{S} \mapsto V=\bigcup_{\mathcal{F} \in \mathcal{S}} \operatorname{supp}_{X}(\mathcal{F})
$$

induce a bijection between

(1) the set of all subsets of the form $V=\bigcup_{i \in \Omega} V_{i}$ with quasicompact open complement $X \backslash V_{i}$ for all $i \in \Omega$, and

(2) the set of all tensor Serre subcategories in $\operatorname{coh}(X)$.

Theorem 42. Let $X$ be a coherent scheme. The assignments

$$
\mathcal{T} \mapsto \mathcal{S}=\left\{\mathcal{F} \in \operatorname{coh}(X) \mid \operatorname{supp}_{X}(\mathcal{F}) \subseteq \bigcup_{n \in \mathbb{Z}, E \in \mathcal{T}} \operatorname{supp}_{X}\left(H_{n}(E)\right)\right\}
$$

and

$$
\mathcal{S} \mapsto\left\{E \in \mathcal{D}_{\text {per }}(X) \mid H_{n}(E) \in \mathcal{S} \text { for all } n \in \mathbb{Z}\right\}
$$

induce a bijection between

(1) the set of all tensor thick subcategories of $\mathcal{D}_{\text {per }}(X)$, and

(2) the set of all tensor Serre subcategories in $\operatorname{coh}(X)$.

Let $X$ be a coherent scheme. The ringed space $\left(\operatorname{Spec}(\operatorname{coh}(X)), \mathcal{O}_{\operatorname{coh}(X)}\right)$ is introduced in the same way as $\left(\operatorname{Spec}(\mathrm{Q} \operatorname{coh}(X)), \mathcal{O}_{\mathrm{Qcoh}(X)}\right)$. 
Theorem (Reconstruction). Let $X$ be a coherent scheme. Then there is a natural isomorphism of ringed spaces

$$
f:\left(X, \mathcal{O}_{X}\right) \stackrel{\sim}{\longrightarrow}\left(\operatorname{Spec}(\operatorname{coh}(X)), \mathcal{O}_{\operatorname{coh}(X)}\right) .
$$

The above theorems are direct consequences of the corresponding theorems for quasicompact, quasiseparated schemes and Theorem [39. The interested reader can check these theorems without difficulty.

\section{REFERENCES}

[1] P. Gabriel, Des catégories abéliennes, Bull. Soc. Math. France 90 (1962), 323-448. MR0232821 $(38: 1144)$

[2] A. B. Buan, H. Krause, and Ø. Solberg, Support varieties: an ideal approach, Homology, Homotopy Appl. 9 (2007), 45-74. MR.2280286 (2008i:18007)

[3] G. Garkusha and M. Prest, Classifying Serre subcategories of finitely presented modules, Proc. Amer. Math. Soc. 136 (2008), 761-770. MR2361847(2008i:13011)

[4] _ Reconstructing projective schemes from Serre subcategories, J. Algebra 319 (2008), 11321153. MR 2379094 (2008k:14002)

[5] _ Torsion classes of finite type and spectra, K-Theory and Noncommutative Geometry, Europ. Math. Soc., Zürich, 2008, pp. 393-412. MR2513341

[6] R. W. Thomason, The classification of triangulated subcategories, Compositio Math. 105 (1997), 1-27. MR 1436741 (98b:18017)

[7] M. J. Hopkins, Global methods in homotopy theory, Homotopy Theory (Durham, 1985), London Math. Soc. Lecture Note Ser., vol. 117, Cambridge Univ. Press, Cambridge, 1987, pp. 73-96. MR 0932260 (89g:55022)

[8] A. Neeman, The chromatic tower for $D(R)$, Topology 31 (1992), 519-532. MR1174255|(93h:18018)

[9] P. Balmer, Presheaves of triangulated categories and reconstruction of schemes, Math. Ann. $\mathbf{3 2 4}$ (2002), 557-580. MR1938458 (2003j:18016)

[10] M. Hovey, Classifying subcategories of modules, Trans. Amer. Math. Soc. 353 (2001), 3181-3191. MR:1828603 (2002i:13007)

[11] A. L. Rosenberg, The spectrum of abelian categories and reconstruction of schemes, Rings, Hopf Algebras, and Brauer Groups (Antwerp/Brussels, 1996), Lecture Notes in Pure and Appl. Math., vol. 197, Marcel Dekker, New York, 1998, pp. 257-274. MR 1615928 (99d:18011)

[12] I. Herzog, The Ziegler spectrum of a locally coherent Grothendieck category, Proc. London Math. Soc. (3) 74 (1997), 503-558. MR1434441 (98j:18017)

[13] H. Krause, The spectrum of a locally coherent category, J. Pure Appl. Algebra 114 (1997), 259-271. MR 1426488 (98e:18006)

[14] E. Enochs and S. Estrada, Relative homological algebra in the category of quasi-coherent sheaves, Adv. Math. 194 (2005), 284-295. MR2139915 (2006a:16012)

[15] A. Grothendieck and J. A. Dieudonné, Eléments de géométrie algébrique. I, Grundlehren Math. Wiss., Bd. 166, Springer-Verlag, Berlin, 1971.

[16] R. Hartshorne, Algebraic geometry, Grad. Texts in Math., No. 52, Springer-Verlag, New YorkHeidelberg, 1977. MR0463157 (57:3116)

[17] G. A. Garkusha, Grothendieck categories, Algebra i Analiz 13 (2001), no. 2, 1-68; English transl., St. Petersburg Math. J. 13 (2002), no. 2, 149-200. MR.1834859 (2003b:18007)

[18] D. Murfet, Modules over a scheme, therisingsea.org.

[19] M. Ziegler, Model theory of modules, Ann. Pure Appl. Logic 26 (1984), 149-213. MR0739577 (86c:03034)

[20] M. Kashiwara and P. Schapira, Sheaves on manifolds, Grundlehren Math. Wiss., Bd. 292, SpringerVerlag, Berlin, 1990. MR1074006 (92a:58132)

[21] J. Lipman, Notes on derived categories and derived functors, www.math.purdue.edu/ ${ }^{1 \text { ipman. }}$

[22] A. Bondal and M. van den Bergh, Generators and representability of functors in commutative and noncommutative geometry, Moscow Math. J. 3 (2003), no. 1, 1-36. MR.1996800 (2004h:18009)

[23] M. Hochster, Prime ideal structure in commutative rings, Trans. Amer. Math. Soc. 142 (1969), 43-60. MR0251026 (40:4257)

[24] P. Balmer, The spectrum of prime ideals in tensor triangulated categories, J. Reine Angew. Math. 588 (2005), 149-168. MR 2196732 (2007b:18012)

[25] M. Prest, The Zariski spectrum of the category of finitely presented modules, Preprint, maths.man.ac.uk/ mprest. 
[26] P. T. Johnstone, Stone spaces, Cambridge Stud. in Adv. Math., vol. 3, Cambridge Univ. Press, Cambridge, 1982. MR0698074 (85f:54002)

[27] J.-P. Soublin, Anneaux et modules cohérents, J. Algebra 15 (1970), 455-472. MR0260799(41:5422)

Department of Mathematics, Swansea University, Singleton Park, SA2 8PP Swansea, United KINGDOM

E-mail address: G.Garkusha@swansea.ac.uk

$U R L:$ http://www-maths.swan.ac.uk/staff/gg

Received 20/JUL/2008

Translated by THE AUTHOR 